\title{
ANGULAR MOMENTUM TRANSPORT IN SOLAR-TYPE STARS: TESTING THE TIMESCALE FOR CORE-ENVELOPE COUPLING
}

\author{
Pavel A. Denissenkov ${ }^{1}$, Marc Pinsonneault, Donald M. Terndrup, and Grant Newsham \\ Department of Astronomy, The Ohio State University, 4055 McPherson Laboratory, 140 West 18th Avenue, Columbus, OH 43210, USA; \\ dpa@ astronomy.ohio-state.edu, pinsono@ astronomy.ohio-state.edu, terndrup@ astronomy.ohio-state.edu, newshamg@astronomy.ohio-state.edu \\ Received 2009 November 5; accepted 2010 May 4; published 2010 May 28
}

\begin{abstract}
We critically examine the constraints on internal angular momentum transport which can be inferred from the spin-down of open cluster stars. The rotation distribution inferred from rotation velocities and periods is consistent for larger and more recent samples, but smaller samples of rotation periods appear biased toward shorter periods relative to $v \sin i$ studies. We therefore focus on whether the rotation period distributions observed in star forming regions can be evolved into the observed ones in the Pleiades, NGC 2516, M 34, M 35, M 37, and M 50 with plausible assumptions about star-disk coupling and angular momentum loss from magnetized solar-like winds. Solid-body (SB) models are consistent with the data for low-mass fully convective stars but highly inconsistent for higher mass stars where the surface convection zone can decouple for angular momentum purposes from the radiative interior. The Tayler-Spruit magnetic angular momentum transport mechanism, commonly employed in models of high-mass stars, predicts SB rotation on extremely short timescales of less than $1 \mathrm{Myr}$ and is therefore unlikely to operate in solar-type pre-main-sequence (pre-MS) and MS stars at the predicted rate. Models with core-envelope decoupling can explain the spin-down of 1.0 and 0.8 solar mass slow rotators with characteristic coupling timescales of $55 \pm 25 \mathrm{Myr}$ and $175 \pm 25 \mathrm{Myr}$, respectively. The upper envelope of the rotation distribution is more strongly coupled than the lower envelope of the rotation distribution, in accord with theoretical predictions that the angular momentum transport timescale should be shorter for more rapidly rotating stars. Constraints imposed by the solar rotation curve are also discussed. We argue that neither hydrodynamic mechanisms nor our revised and less efficient prescription for the Tayler-Spruit dynamo can reproduce both spin-down and the internal solar rotation profile by themselves. It is likely that a successful model of angular momentum evolution will involve more than one mechanism. Further observational studies, especially of clusters younger than $100 \mathrm{Myr}$, will provide important additional constraints on the internal rotation of stars and could firmly rule out or confirm the operation of major classes of theoretical mechanisms.
\end{abstract}

Key words: stars: evolution - stars: interiors - Sun: rotation

Online-only material: color figures

\section{INTRODUCTION}

Rotation is an important attribute of the life of a star. When it is fast enough, rotation can trigger various (magneto-) hydrodynamic instabilities that will drive mixing and angular momentum transport in the star (e.g., Zahn 1992; Spruit 1999). Unfortunately, except for the Sun, observations give information only about surface rotation of stars. Therefore, indirect methods have to be used to study rotation-driven transport processes in stellar interiors. When stars reach the main sequence (MS), their surface response to the torque from a magnetized wind depends on the timescale for internal angular momentum transport. If open clusters are treated as an evolutionary sequence, the assumptions of solid body (SB) and differential rotation (DR) lead to statistically distinguishable differences in the time evolution of the distributions of cluster star rotation rates (see, for example, Keppens et al. 1995; Krishnamurthi et al. 1997; Allain 1998). At late ages, models also have to be consistent with the strong coupling evident in the solar internal rotation profile, with nearly SB rotation in the radiative core down to $\sim 0.2 R_{\odot}$ (Tomczyk et al. 1995; Couvidat et al. 2003).

The prior estimates of the timescale for core-envelope coupling in solar-type stars usually agreed on a short coupling time of order $1 \mathrm{Myr}$ for the fastest rotators. However, they consid-

\footnotetext{
1 Current address: Department of Physics, and Astronomy, University of Victoria, P.O. Box 3055, Victoria, B.C., V8W 3P6, Canada.
}

erably disagreed on the coupling time for slowly rotating solar analogs for which the estimated values varied from 10-100 Myr (e.g., Keppens et al. 1995; Allain 1998) to 0.5 Gyr (e.g., Irwin et al. 2007). This discrepancy has been caused, on the one hand, by not carefully treating the selection effects and statistics and, on the other hand, by making particular assumptions about such things as the disk-locking time, initial rotation period distribution, or the relative cluster ages which have since been updated. For example, the early work assumed that $\alpha$ Per was $50 \mathrm{Myr}$ and the Pleiades was $70 \mathrm{Myr}$ old (MacGregor \& Brenner 1991). In this work, we apply rigorous methods of statistical analysis to the most recent large observational data sets on rotation periods of low-mass stars in open clusters and extensive computational modeling of their rotational evolution to constrain the timescale for core-envelope coupling in the slowest rotators.

In this paper, we find that the timescale for core-envelope coupling depends on both rotation rate and mass, but it is significantly longer than would be predicted if SB rotation was enforced on a short timescale. Angular momentum transport by magnetic torques generated by the Tayler-Spruit dynamo (Spruit 1999, 2002) does not pass this key test because it always enforces SB rotation in a solar-type star, no matter how slowly it rotates. This disagrees with the observational evidence that slow rotators in young clusters are most likely to possess DR (their cores rotate faster than their envelopes) rather than follow the $P$-age relations computed using SB rotation models. Furthermore, we show that even a revised prescription 
Table 1

Rotation Period Data

\begin{tabular}{|c|c|c|c|c|c|}
\hline \multirow[t]{2}{*}{ Cluster } & \multirow[t]{2}{*}{ Age (Myr) } & \multicolumn{3}{|c|}{ Number of Stars in the Sample } & \multirow[t]{2}{*}{ Source } \\
\hline & & $M / M_{\odot} \leqslant 0.4$ & $0.7 \leqslant M / M_{\odot}<0.9$ & $0.9 \leqslant M / M_{\odot} \leqslant 1.1$ & \\
\hline $\mathrm{ONC}$ & 2 & 32 & 3 & 53 & Rebull et al. (2004) \\
\hline NGC 2264 & 4 & 13 & 12 & 63 & Rebull et al. (2004) \\
\hline Lupus & 4 & 0 & 2 & 12 & Rebull et al. (2004) \\
\hline NGC 2362 & 5 & 70 & 46 & 44 & Irwin et al. (2008a) \\
\hline Tau-Aur & 6 & 0 & 2 & 23 & Rebull et al. (2004) \\
\hline IC $2391 \&$ IC 2602 & 40 & 0 & 8 & 28 & Rebull et al. (2004) \\
\hline$\alpha$ Per & 70 & 0 & 8 & 28 & Rebull et al. (2004) \\
\hline Pleiades & 115 & 0 & 20 & 19 & Rebull et al. (2004) \\
\hline Pleiades $(v \sin i)$ & 115 & 39 & 50 & 51 & Terndrup et al. (2000) \\
\hline M 50 & 130 & 163 & 252 & 62 & Irwin et al. (2009) \\
\hline NGC 2516 & 180 & 183 & 0 & 0 & Irwin et al. (2008a) \\
\hline M 35 & 150 & 0 & 154 & 111 & Meibom et al. (2009) \\
\hline M 37 & 550 & 0 & 197 & 128 & Hartman et al. (2009) \\
\hline Hyades & 600 & 0 & 8 & 17 & Radick et al. (1987); Prosser et al. (1995) \\
\hline
\end{tabular}

for the Tayler-Spruit dynamo proposed by Denissenkov \& Pinsonneault (2007), which reduces the effective magnetic viscosity by nearly 2 orders of magnitude, is still too efficient in redistributing angular momentum in the outer parts of the radiative core; this is inconsistent with the observed evolution of slowly rotating solar analogues in the $P$-age plane. The revised prescription comes to a better agreement with observations when it is supplemented by an additional angular momentum transport mechanism that should be able to operate in the inner core on a longer timescale. Such a mechanism is also needed to assist the revised prescription in shaping the solar SB rotation.

In our work, three different theoretical models are employed to study the rotational evolution of solar-type stars: a simple double-zone model and two full stellar evolutionary models, one with a constant viscosity and the other with the effective magnetic viscosities provided by the original and revised prescriptions for the Tayler-Spruit dynamo. The models are described in detail and compared with each other in Section 2. Because the choice of basic model parameters is necessarily constrained by observations, we find it helpful to briefly introduce the samples of rotation period and $v \sin i$ data, that will be used for a more detailed analysis later in Section 3, already at the beginning of Section 2. In Section 3, we present the results of our computations of rotation period distributions for solartype stars, as they evolve from the pre-MS deuterium birth line to the solar age, and compare them with observations of rotation periods in open clusters. A brief discussion and our main conclusions are given in Section 4.

\section{BASIC THEORETICAL MODELS AND OBSERVATIONAL DATA}

For studying the angular momentum evolution of solar-type stars, it is important to specify correct initial and boundary conditions as well as to incorporate into the stellar evolution code three principal processes that are believed to govern changes of their surface rotation with time: disk-locking, internal transport of angular momentum, and angular momentum loss from the surface. In this section, we briefly describe common ingredients of our rotating stellar evolutionary models, such as the input physics, the initial and boundary conditions, the law used for angular momentum loss, and our considered mechanisms for internal angular momentum transport.
Because of the complex inter-relationship between the physical processes involved in the rotational evolution of solar-type stars, it is also important to use all available observational data that can constrain it. Fortunately, thanks to ongoing and planned ground and space based planet transit searches (such as the Deep MMT Transit Survey, the Monitor Project, Kepler, and COROT), extensive data sets of rotation periods for young and intermediate-age open cluster low-mass stars have been accumulating quickly during the last few years. The data that will be used in our paper to constrain the models are summarized in Table 1. Their corresponding rotation periods are plotted in Figure 1 (crosses) for illustration.

\subsection{Common Model Ingredients}

In our full rotating evolutionary computations, we employ an upgraded version of the computer code used by Denissenkov \& VandenBerg (2003). The most recent update is the adoption of Alan Irwin's improved equation of state (EOS). ${ }^{2}$ In addition, the energy losses due to neutrino emission are now calculated with the code distributed by Itoh et al. (1996). We use OPAL opacities (Rogers \& Iglesias 1992) for temperatures above $\sim 10^{4}$ K, complemented by Alexander \& Ferguson (1994) data for lower temperatures. Nuclear reaction rates are taken from the NACRE compilation (Angulo et al. 1999). Gravitational settling is not included.

We accept Zahn's concept of the rotation-induced anisotropic turbulence in stellar radiative zones, with horizontal components of the turbulent viscosity strongly dominating over those in the vertical direction (Zahn 1992), which assumes that the horizontal turbulence has erased the latitudinal DR. This allows us to consider the angular velocity as a function of radius alone. We take into account small corrections to the stellar structure equations arising from the distortion of equipotential surfaces by such shellular rotation (for details, see Denissenkov \& VandenBerg 2003). We used the Grevesse \& Noels (1993) mixture of heavy elements. Our code has been calibrated to reproduce the solar luminosity $L_{\odot}=3.85 \times 10^{33} \mathrm{erg} \mathrm{s}^{-1}$ and radius $R_{\odot}=6.96 \times 10^{10} \mathrm{~cm}$ at the solar age of $t_{\odot}=4.57 \mathrm{Gyr}$. This procedure yields the initial hydrogen mass fraction $X=$

\footnotetext{
2 We use the EOS code that is made publicly available at http://freeeos.sourceforge.net/ under the GNU General Public License.
} 

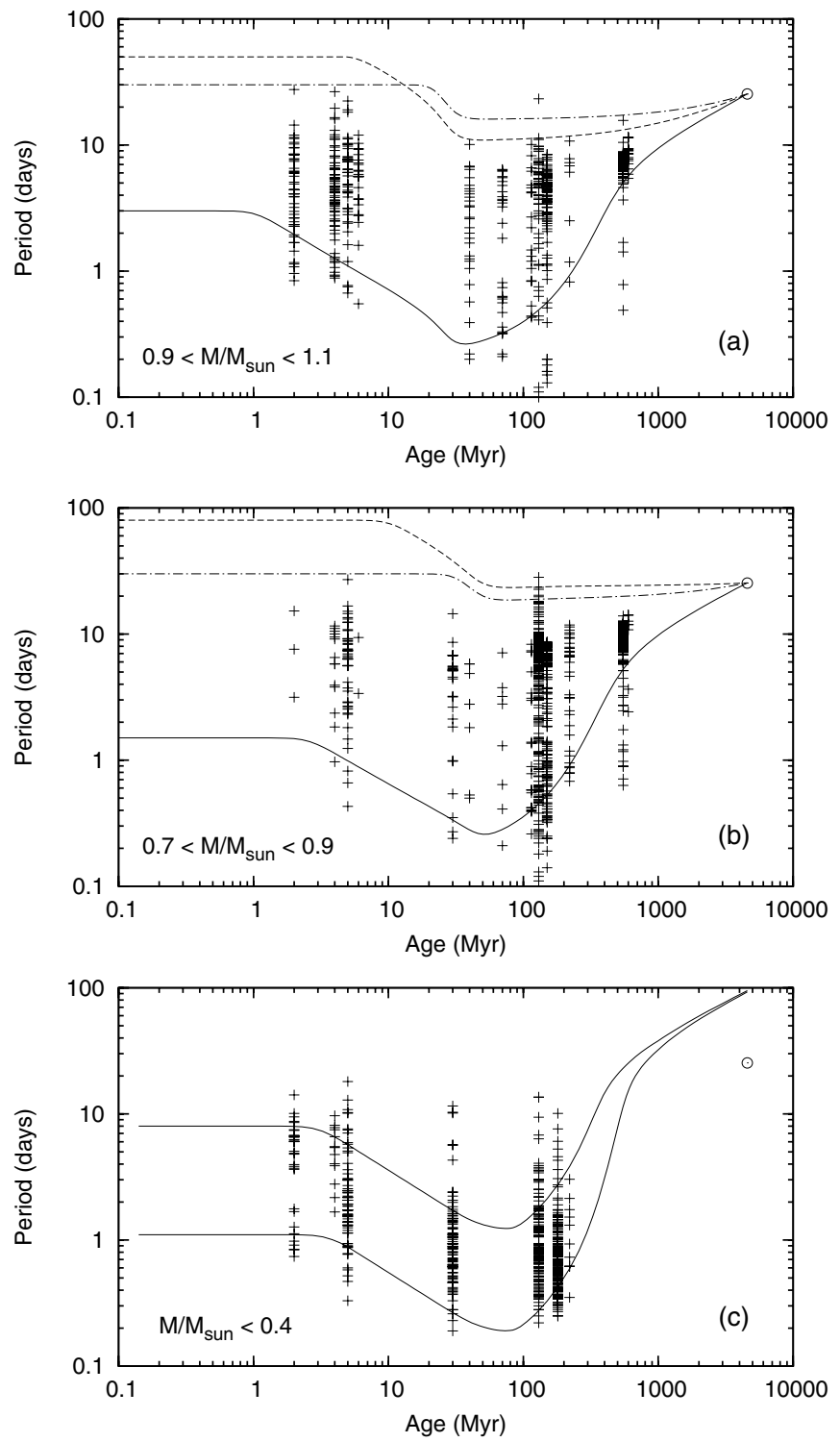

Figure 1. Rotation period data (crosses) for the three mass bins used in this paper (see Table 1). Curves represent the SB rotational evolution computed with different values of the initial period and disk-locking time using the double-zone model and common model ingredients that are described and discussed in the text later.

0.708 for the solar heavy-element mass fraction $Z=0.018$, and a mixing length $\alpha$ of 1.75 .

\subsection{Initial and Boundary Conditions}

Our computations start on the pre-MS deuterium birth line of Palla \& Stahler (1991). For stars with masses $M=1 M_{\odot}$ and $0.8 M_{\odot}$, the estimated birth line radii are $4.8 R_{\odot}$ and $2.9 R_{\odot}$, respectively. We assume that convective regions rotate as solid bodies at all times. Rotation at the birth line is specified by the initial rotation period $P_{0}$. In practice, we begin with a nonrotating fully convective model that is located above the birth line. This model is evolved down to the birth line where it is spun up to $P=P_{0}$, which yields the initial model for our rotating evolutionary computations. The initial period values are taken from the observed period distributions of solar-type stars in the youngest stellar clusters from our compiled data samples: the Orion Nebula cluster (hereafter, ONC or Orion), NGC 2264, and NGC 2362 (Table 1). The Kolmogorov-Smirnov (KS) test gives high probabilities for these distributions to have been drawn from the same real distribution (Figure 2). Because stars still possess deep surface convection zones at these young ages, different ways of achieving the same surface rotation rate at the end of this stage will have similar final outcomes.

During its approach to the zero-age main sequence (ZAMS), the protostar contracts. If its total angular momentum $J_{\text {tot }}$ conserved on the pre-MS then the star would spin up as a result of the contraction. However, observations show that a large number of stars arriving at the ZAMS rotate slowly, as if their angular velocity rather than angular momentum has remained constant (see Figure 3 of Rebull et al. 2004). The most plausible explanation is that the interaction of protostars with their accretion disks extracts angular momentum from the central object, reducing or preventing spin-up as they contract. This can occur from magnetic coupling between the protostar and disk (Koenigl 1991; Shu et al. 1994) or through an enhanced wind (Matt \& Pudritz 2005). As in most other works (e.g., Krishnamurthi et al. 1997; Bouvier et al. 1997; Allain 1998; Tinker et al. 2002), we model the interaction between the protostar and accretion disk in a simple way, assuming that during an initial time interval $0 \leqslant t \leqslant \tau_{\mathrm{d}}$ the interaction maintains $P(t)=P_{0}$. The disk-locking time $\tau_{\mathrm{d}}$ is considered as a free parameter.

In our stellar evolution code, the transport of angular momentum is treated as a diffusion process described by

$$
\frac{d}{d t}\left(r^{2} \Omega\right)=\frac{\partial}{\partial M_{r}}\left[\left(4 \pi r^{2} \rho\right)^{2} r^{2} v \frac{\partial \Omega}{\partial M_{r}}\right],
$$

where $d / d t$ is a derivative taken at a constant $M_{r}$, and $v$ is a viscosity whose physical nature has yet to be identified in real stars. Equation (1) needs two boundary conditions. A natural initial condition for it is that the fully convective birth line model rotates as an SB with $\Omega\left(0, M_{r}\right)=\Omega_{\mathrm{e}}(0)=2 \pi / P_{0}$, where $\Omega_{\mathrm{e}}=2 \pi / P$ is the angular velocity of star's convective envelope. The inner SB rotation boundary condition $\partial \Omega / \partial M_{r}=0$ is applied at the surface of the innermost mass shell used in our computations, at $M_{r} \approx 0$. To derive the outer boundary condition that has to be applied at the bottom of convective envelope $M_{r}=M_{\text {bce }}$, we integrate Equation (1) from $M_{r}=0$ to $M_{r}=M_{\mathrm{bce}}$, taking into account that the time derivative of the angular momentum of radiative core is

$$
\begin{aligned}
\dot{J}_{\mathrm{c}}= & \frac{d}{d t} \frac{2}{3} \int_{0}^{M_{\mathrm{bce}}} r^{2} \Omega d M_{r}=\frac{2}{3} r_{\mathrm{bce}}^{2} \Omega_{\mathrm{e}} \dot{M}_{\mathrm{bce}} \\
& +\frac{2}{3} \int_{0}^{M_{\mathrm{bce}}} \frac{d}{d t}\left(r^{2} \Omega\right) d M_{r}=\dot{J}_{\mathrm{tot}}-\dot{J}_{\mathrm{e}} .
\end{aligned}
$$

Here, $J_{\mathrm{e}}=I_{\mathrm{e}} \Omega_{\mathrm{e}}$ is the angular momentum of convective envelope, $I_{\mathrm{e}}$ being the envelope's moment of inertia. Finally, we obtain the following upper boundary condition:

$$
\begin{aligned}
I_{\mathrm{e}} \dot{\Omega}_{\mathrm{e}}= & \dot{J}_{\text {tot }}-\dot{I}_{\mathrm{e}} \Omega_{\mathrm{e}}-\frac{2}{3} r_{\text {bce }}^{2} \Omega_{\mathrm{e}} \dot{M}_{\text {bce }} \\
& -\frac{2}{3}\left[\left(4 \pi r^{2} \rho\right)^{2} r^{2} v \frac{\partial \Omega}{\partial M_{r}}\right]_{M_{\text {bce }}},
\end{aligned}
$$

in which $\dot{J}_{\text {tot }}$ is the rate of angular momentum loss from the stellar surface.

\subsection{Angular Momentum Loss and Internal Transport}

For the angular momentum loss from the surface, we adopt the magnetized stellar wind prescription of Krishnamurthi et al. 

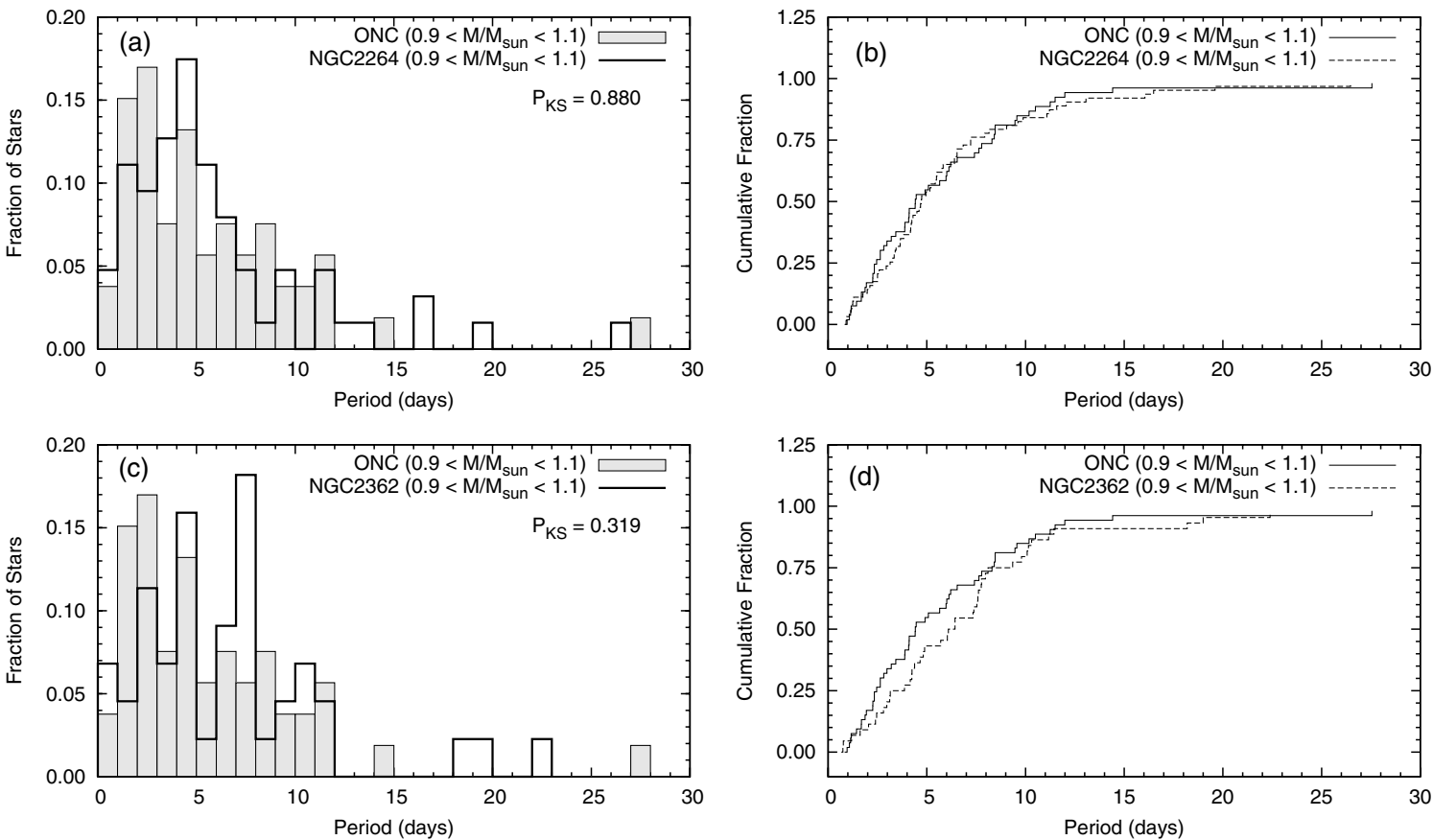

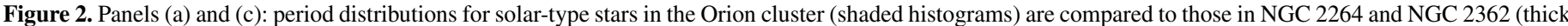

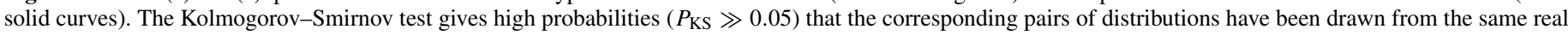
distribution. Panels (b) and (d): cumulative distribution functions for the two pairs of clusters.

(1997):

$$
\dot{J}_{\mathrm{tot}}=-K_{\mathrm{w}} \sqrt{\frac{R / R_{\odot}}{M / M_{\odot}}} \min \left(\Omega_{\mathrm{e}} \Omega_{\mathrm{sat}}^{2}, \Omega_{\mathrm{e}}^{3}\right) .
$$

Here, $\Omega_{\text {sat }}$ is the velocity at which the wind is saturated. The parameter $\Omega_{\text {sat }}$ is known to strongly depend on the stellar mass (e.g., Andronov et al. 2003). In Figure 3, we have used upper 90th percentiles of the $\Omega_{\mathrm{e}}$ distributions for our cluster sample to adjust the appropriate values of $\Omega_{\text {sat }}$ for the three mass bins that we use in this study. For internal angular momentum transport, we assume that fast rotators behave as SB rotators, a result both of theoretical calculations (Krishnamurthi et al. 1997; Sills et al. 2000) and indicated by prior spin-down studies (Irwin et al. 2007; there were prior claims by the Monitor group). The error bars to the percentiles were calculated using bootstrap simulations by generating 1000 sampling distributions for each cluster. Our adjusted $\Omega_{\text {sat }}$ values (shown in each panel in Figure 3) are close to those reported by Andronov et al. (2003). In particular, we have found that the same value of $\Omega_{\text {sat }}=8 \Omega_{\odot}$ can be used for either of our "solar-type" mass bins, $0.7 \leqslant M / M_{\odot}<0.9$ and $0.9 \leqslant M / M_{\odot} \leqslant 1.1$, to reproduce reasonably well their corresponding 90 th percentiles. This value lies between the estimates $\Omega_{\text {sat }}=6.4 \Omega_{\odot}$ and $\Omega_{\text {sat }}=10.5 \Omega_{\odot}$ obtained by Andronov et al. (2003) for stars with $M=0.8 M_{\odot}$ and $M=1.0 M_{\odot}$, respectively. For the "fully convective" $M \leqslant 0.4 M_{\odot}$ bin, we have adjusted the parameter $\Omega_{\text {sat }}=2.5 \Omega_{\odot}$. It can be compared with the value of $1.8 \Omega_{\odot}$ used by Andronov et al. (2003) as a saturation threshold for their $0.4 M_{\odot}$ model star. Note that in all our computations we use stellar models with masses appropriate for the considered mass bins, namely, $0.3 M_{\odot}, 0.8 M_{\odot}$, and $1.0 M_{\odot}$. The parameter $K_{\mathrm{W}}$ in Equation (3) is calibrated by requiring that our $0.8 M_{\odot}$ and $1.0 M_{\odot}$ models have $\Omega_{\mathrm{e}}=\Omega_{\odot}=2.86 \times 10^{-6} \mathrm{rad} \mathrm{s}^{-1}$ ( $P_{\odot}=25.4$ days) at the solar age. For stars with $M \lesssim 0.4 M_{\odot}$, we use our $0.3 M_{\odot}$ model and the solar calibrated value of
$K_{\mathrm{w}} \approx 3.19 \times 10^{47} \mathrm{~cm}^{2} \mathrm{~g} \mathrm{~s}^{-2}$ that has produced the solid curve in Figure 3(a).

\subsubsection{Constant Viscosity}

In this section, we consider angular momentum transport in rotating stellar evolutionary models with a constant viscosity $v\left(t, M_{r}\right)=v_{0}$. Although the assumption of constant viscosity does not reveal the physical mechanism responsible for the transport of angular momentum in solar-type stars, it nevertheless permits us to estimate both an instructive minimum value $\left(v_{0}\right)_{\min }$ that still results in the solar SB rotation and a maximum value $\left(v_{0}\right)_{\max }$ such that the rotational evolution with any value in excess of it looks identical to that with $v_{0}=\left(v_{0}\right)_{\max }$. We have computed the evolution of a rotating $1 M_{\odot}$ star for two combinations of the disk-locking time (in Myr) and initial rotation period (in days): $\left(\tau_{\mathrm{d}}, P_{0}\right)=(6,8)$ and $(2,3)$. For either of these combinations, the computations have been done for the same set of values of $\Omega_{\text {sat }}=8 \Omega_{\odot}$ and $v_{0}=2.5 \times 10^{4}, 5 \times 10^{4}, 10^{5}, 2.5 \times 10^{5}, 5 \times 10^{5}, 10^{6}, 10^{7}$, and $10^{8} \mathrm{~cm}^{2} \mathrm{~s}^{-1}$. Results are presented in Figure 4 with solid and dashed curves for the first and second combination of initial conditions, respectively. Models with $v_{0} \leqslant\left(v_{0}\right)_{\min } \approx$ $5 \times 10^{4} \mathrm{~cm}^{2} \mathrm{~s}^{-1}$ have a residual DR at the solar age inconsistent with helioseismic data. On the other hand, the rotational evolution of models with $v_{0} \geqslant\left(v_{0}\right)_{\max } \approx 10^{6} \mathrm{~cm}^{2} \mathrm{~s}^{-1}$ is almost indistinguishable from one another.

\subsubsection{Effective Magnetic Viscosities from the Tayler-Spruit Dynamo}

Spruit (1999, 2002) has elaborated upon the finding by Tayler (1973) that toroidal magnetic fields frozen into plasma in a stellar radiative zone are always subject to a pinch-type instability. A release of magnetic energy by this instability causes concentric magnetic rings to slide sideways, mainly horizontally, along the equipotential surfaces and, to some extent, also along the radius. Magnetic induction makes it 

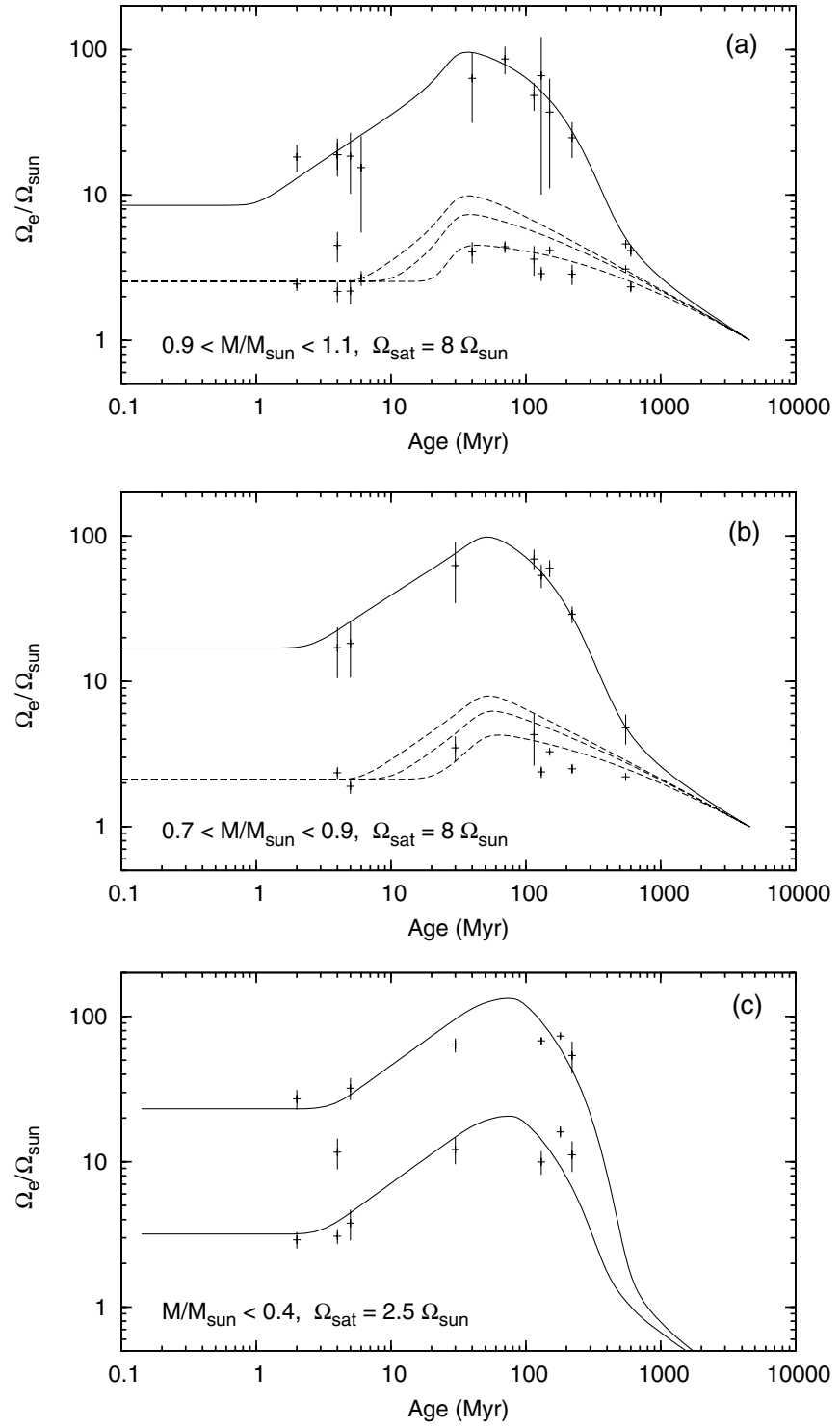

Figure 3. Upper 90th and lower 10th percentiles of $\Omega_{\mathrm{e}}=2 \pi / P$ distributions (crosses) for all of our compiled data samples (Table 1). The 90th percentiles are used to adjust the parameter $\Omega_{\text {sat }}$ (shown in each panel) by fitting them to the SB rotation evolution (solid curves). The 10th percentiles for the fully convective stars are also fitted well by an SB rotation evolution curve (panel (c)). The percentile vertical error bars were evaluated through bootstrap simulations.

possible for the unstable radial displacement to produce a weak poloidal field $B_{r}$ at the expense of toroidal magnetic field $B_{\varphi}$. If the radiative zone rotates differentially, the poloidal field can be stretched around the rotation axis into a new toroidal field that will again be subject to the Tayler instability. Spruit's original idea was that these consecutive poloidal/ toroidal field generations might sustain each other under certain circumstances, thus forming a dynamo loop. The Tayler-Spruit dynamo could drive some mixing through unstable radial displacements with an effective diffusion coefficient $\eta_{\mathrm{e}}$, as well as some angular momentum transport by magnetic torques proportional to the product $B_{r} B_{\varphi}$ with an effective viscosity $v_{\mathrm{e}}$. However, estimating the diffusion coefficients is more challenging. Denissenkov \& Pinsonneault (2007) argued that Spruit overestimated $\eta_{\mathrm{e}}$ and $v_{\mathrm{e}}$ because the effective horizontal displacement is reduced away from the rotation axis; the heuristic approach used to derive the diffusion coefficients was

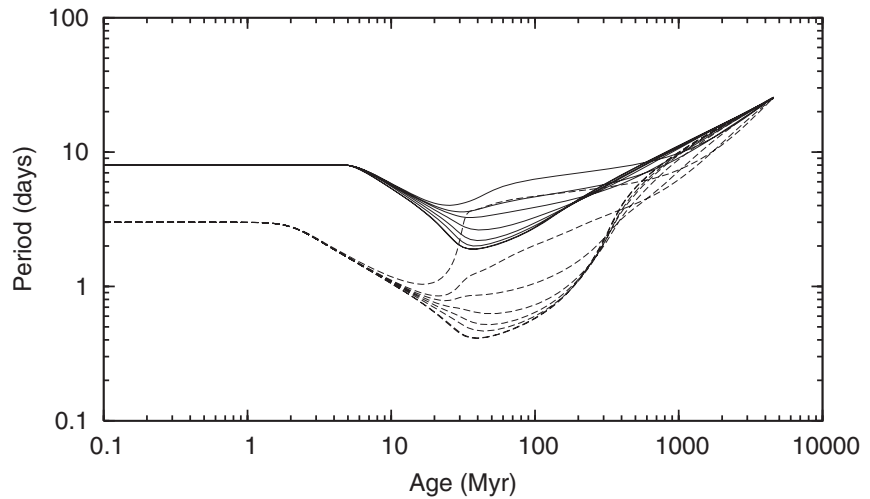

Figure 4. Rotation period evolution of the Sun computed using the constant viscosity model for the initial conditions $\left(\tau_{\mathrm{d}}, P_{0}\right)=(6 \mathrm{Myr}, 8$ days) (solid curves), and ( $\left.\tau_{\mathrm{d}}, P_{0}\right)=$ (2 Myr, 3 days) (dashed curves), and for the values of $v_{0}=2.5 \times 10^{4}, 5 \times 10^{4}, 10^{5}, 2.5 \times 10^{5}, 5 \times 10^{5}, 10^{6}, 10^{7}$, and $10^{8} \mathrm{~cm}^{2} \mathrm{~s}^{-1}$ (from upper to lower curve for either combination of initial conditions).

also critiqued in Section 4 of Zahn et al. (2007). The latter authors also did not find the predicted dynamo mechanism in their numerical simulation; see also Rüdiger et al. (2009).

Whereas the original prescription for the Tayler-Spruit dynamo has been shown to produce a solar rotation profile in agreement with helioseismic data (Eggenberger et al. 2005), the revised prescription fails to do so alone (Denissenkov \& Pinsonneault 2007). One of the objectives of the present work is to see if these prescriptions can yield the evolution of surface rotation consistent with the rotation period data for solar-type stars in open clusters.

Let us summarize the basic equations for the magnetic transport coefficients in the Spruit mechanism. For details, the reader is referred to the paper of Denissenkov \& Pinsonneault (2007). For Spruit's original prescription, it is convenient to present the effective magnetic diffusivity and viscosity in the following forms:

$$
\eta_{\mathrm{e}}=\alpha K y^{3}, \quad \text { and } \quad v_{\mathrm{e}}=\alpha K \frac{N_{T}^{2}+N_{\mu}^{2}}{\Omega^{2} q^{2}} y^{2} .
$$

Here, $q=|\partial \ln \Omega / \partial \ln r|$ is the rotational shear, and $y$ is a solution of the fourth-order algebraic equation

$$
\alpha y^{4}-\alpha y^{3}+\beta y-2=0,
$$

where

$$
\alpha=r^{2} \frac{\Omega^{7} q^{4}}{K\left(N_{T}^{2}+N_{\mu}^{2}\right)^{3}}, \text { and } \beta=2 \frac{N_{\mu}^{2}}{N_{T}^{2}+N_{\mu}^{2}}
$$

are dimensionless coefficients. In Equations (4) and (6), we have used standard notations for the thermal diffusivity

$$
K=\frac{4 a c T^{3}}{3 \kappa \rho^{2} C_{P}},
$$

where $\kappa$ and $C_{P}$ represent the opacity and the specific heat at constant pressure, respectively, and for the $T$ - and $\mu$-component of the square of the Brunt-Väisäla (buoyancy) frequency

$$
N_{T}^{2}=\frac{g \delta}{H_{P}}\left(\nabla_{\mathrm{ad}}-\nabla_{\mathrm{rad}}\right) \text {, and } N_{\mu}^{2}=g \varphi\left|\frac{\partial \ln \mu}{\partial r}\right| .
$$

In the last expressions, $g$ is the local gravity, $H_{P}$ is the pressure scale height, $\nabla_{\mathrm{ad}}$ and $\nabla_{\mathrm{rad}}$ are the adiabatic and radiative 
temperature gradients (logarithmic and with respect to pressure), and $\mu$ is the mean molecular weight. The quantities $\delta=$ $-(\partial \ln \rho / \partial \ln T)_{P, \mu}$ and $\varphi=(\partial \ln \rho / \partial \ln \mu)_{P, T}$ are determined by the EOS. In particular, for the perfect gas law $\delta=\varphi=1$.

When using the effective magnetic viscosity $v=v_{\mathrm{e}}$ in the angular momentum transport Equation (1), it is important to remember that the Tayler-Spruit dynamo keeps operating only as long as the effective diffusivity $\eta_{\mathrm{e}}$ remains larger than the magnetic diffusivity $\eta_{\text {mag. }}$. When $\eta_{\mathrm{e}}$ approaches $\eta_{\text {mag }}$ the poloidal magnetic field decays through Ohmic dissipation faster than it is generated by the unstable radial displacements of magnetic rings. Although theory says nothing about the behavior of $v_{\mathrm{e}}$ in this limit, we can formally estimate a minimum value of the effective magnetic viscosity that is reached when $\eta_{\mathrm{e}}=\eta_{\text {mag }}$. To do this, we replace $\eta_{\mathrm{e}}$ with $\eta_{\text {mag }}$ in the first of Equations (4) and then solve Equations (4)-(6) with respect to $q$ and $v_{\mathrm{e}}$. The effective magnetic viscosity approaches its minimum value of

$$
\begin{aligned}
\left(v_{\mathrm{e}}\right)_{\min }= & 6.96 \times 10^{7}\left(\frac{r}{R_{\odot}}\right)\left(\frac{2+\varepsilon}{\beta+\varepsilon}\right)^{1 / 2}\left(\frac{\eta_{\mathrm{mag}}}{10^{3}}\right)^{1 / 2} \\
& \times\left(\frac{N_{T}^{2}+N_{\mu}^{2}}{10^{-6}}\right)^{-1 / 2}\left(\frac{\Omega}{10^{-5}}\right)^{3 / 2} \mathrm{~cm}^{2} \mathrm{~s}^{-1}
\end{aligned}
$$

when the shear is reduced to

$$
\begin{aligned}
q_{\min }= & 0.379\left(\frac{r}{R_{\odot}}\right)^{-1 / 2}\left(\frac{\beta+\varepsilon}{2+\varepsilon}\right)^{3 / 4}\left(\frac{\eta_{\mathrm{mag}}}{10^{3}}\right)^{1 / 4} \\
& \times\left(\frac{N_{T}^{2}+N_{\mu}^{2}}{10^{-6}}\right)^{3 / 4}\left(\frac{\Omega}{10^{-5}}\right)^{-7 / 4} .
\end{aligned}
$$

The normalizations in the last two equations, for which we have used values typical for the solar interior, assume that all quantities are expressed in cgs units. We have also introduced the reciprocal of the Roberts number $\varepsilon=\eta_{\mathrm{mag}} / K \ll 1$.

The ratio $\beta$ defined by the second of Equations (6) remains small $(\beta \ll 1)$ everywhere in the star until the age of $\sim 30 \mathrm{Myr}$ because nuclear reactions have not yet built up a sufficiently strong $\mu$-gradient in the stellar core. Given that by this age $\Omega_{\mathrm{e}}$ approaches its maximum value that turns out to exceed $\sim 3 \Omega_{\odot} \approx 10^{-5} \mathrm{rad} \mathrm{s}^{-1}$ in most interesting cases (see Section 3, and panel (a) in Figure 3), it is obvious from Equation (8) that $\left(v_{\mathrm{e}}\right)_{\min }$ will be of order $10^{8} \mathrm{~cm}^{2} \mathrm{~s}^{-1}$ everywhere in our model star during the first tens Myr of its evolution. Furthermore, since $\beta$ continues to remain very small outside the radius $r \sim 0.2 R_{\odot}$, where $N_{\mu}^{2} \ll N_{T}^{2}$, up to the solar age, we can expect that $\left(v_{\mathrm{e}}\right)_{\text {min }}$ will keep values of order $10^{6}-10^{8}$ in the outer part of the radiative core even at older ages. These expectations are confirmed by our detailed computations. Taking into account that Equation (8) gives only a lower limit for the effective magnetic viscosity and the inner core with $r \lesssim 0.2 R_{\odot}$ contributes less than $10 \%$ to the total moment of inertia of the radiative core, we anticipate that Spruit's original prescription should always bring about the $P$-age relations characteristic of SB rotators, like those obtained with our constant viscosity model for $v_{0}>\left(v_{0}\right)_{\max }$. However, we have to ensure that in our computations the viscosity declines abruptly as soon as the shear $q$ is reduced below its critical value given by Equation (9). This requirement may leave some residual DR in the core of our final solar model.

In our full rotating stellar evolutionary models, we have used an approximate computational method. It assumes that, as soon as a radiative core develops in a pre-MS star and

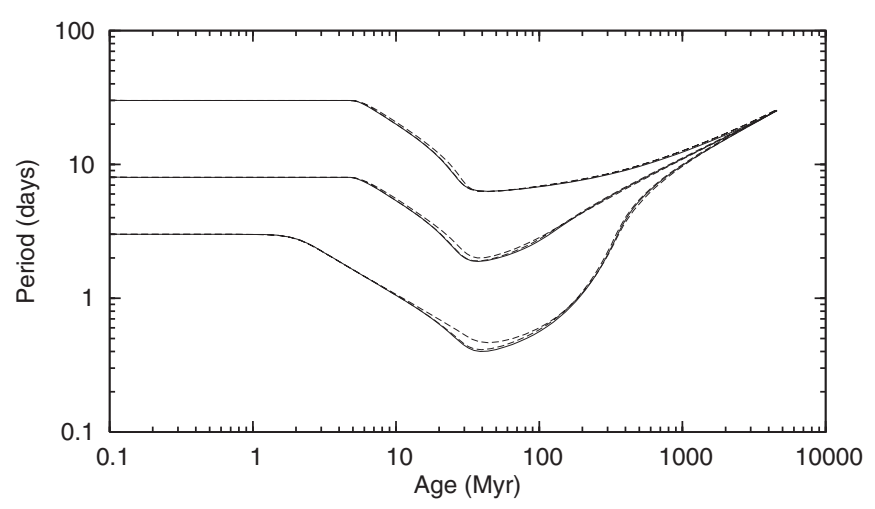

Figure 5. Rotation period evolution computed for the initial conditions $\left(\tau_{\mathrm{d}}, P_{0}\right)=(6 \mathrm{Myr}, 8$ days $),(6 \mathrm{Myr}, 30$ days $)$, and ( $2 \mathrm{Myr}, 3$ days) using the original prescription for the Tayler-Spruit dynamo (solid curves) and the constant viscosity model with $\nu_{0}=10^{6}$, and $10^{7} \mathrm{~cm}^{2} \mathrm{~s}^{-1}$ (dashed curves). The dashed curves for $v_{0}=10^{7} \mathrm{~cm}^{2} \mathrm{~s}^{-1}$ almost coincide with the solid curves. All computations have been performed with $M=1 M_{\odot}$ and $\Omega_{\text {sat }}=8 \Omega_{\odot}$.

its rotation profile begins to deviate from uniform one, the effective magnetic viscosity is large enough everywhere in the core to potentially restore its uniform rotation. Our test computations confirm this. However, when the redistribution of angular momentum by magnetic torques has led to $q \approx q_{\min }$, the Tayler-Spruit dynamo ceases to work, and its related viscosity should be replaced with the molecular one $v_{\mathrm{mol}} \ll\left(v_{\mathrm{e}}\right)_{\min }$. As we have noted, theory does not describe how this transition from $\left(v_{\mathrm{e}}\right)_{\text {min }}$ to $v_{\text {mol }}$ occurs. When an increase of $\Omega_{\mathrm{e}}$ at $t>\tau_{\mathrm{d}}$ caused by the residual pre-MS contraction of the star and its angular momentum conservation finally gives way to an $\Omega_{\mathrm{e}}$ decrease due to the surface loss of angular momentum with the magnetized stellar wind, a much stronger DR tends to accumulate in the core. However, as soon as the shear exceeds its critical value $q_{\min }$, a large viscosity of order $\left(v_{\mathrm{e}}\right)_{\min }$ will resume the redistribution of angular momentum by magnetic torques smoothing out the angular velocity gradient until $q$ drops below $q_{\min }$ again. Following this qualitative picture, we put into Equation (1) $v=\left(v_{\mathrm{e}}\right)_{\min }$ multiplied by an exponential factor that cancels $v$ when $q$ approaches $q_{\min }$.

Figure 5 compares the results of our computations using this method for $\left(\tau_{\mathrm{d}}, P_{0}\right)=(6,8),(6,30)$, and $(2,3)$ (solid curves) with results that we obtained for the same initial conditions but applying the constant viscosities $v_{0}=10^{6}$ and $10^{7} \mathrm{~cm}^{2} \mathrm{~s}^{-1}$ (dashed curves). We have $\Omega_{\text {sat }}=8 \Omega_{\odot}$ throughout. The comparison shows that, for Spruit's original prescription, the rotation period evolution of the Sun coincides with that of $v_{0}=10^{7} \mathrm{~cm}^{2} \mathrm{~s}^{-1}>\left(v_{0}\right)_{\max }$; i.e., it always corresponds to the $\mathrm{SB}$ rotation case. However, the solid curve in the bottom panel in Figure 6 demonstrates that, unlike the constant viscosity model with $v_{0}>\left(v_{0}\right)_{\max }$, our solar model computed using Spruit's original prescription does contain a small differentially rotating core, as we expected. Unfortunately, its presence cannot be revealed by available helioseismic data (see, however, García et al. 2007). This result agrees with that reported by Eggenberger et al. (2005).

For Spruit's revised prescription (Denissenkov \& Pinsonneault 2007), the effective magnetic diffusivity and viscosity are given by the following equations:

$$
\eta_{\mathrm{e}}=2 K \frac{\Omega^{2} q^{2}-N_{\mu}^{2}}{N_{T}^{2}+N_{\mu}^{2}-\Omega^{2} q^{2}}, \text { and } v_{\mathrm{e}}=\left(\frac{r^{2} \Omega \eta_{\mathrm{e}}^{2}}{q^{2}}\right)^{1 / 3} .
$$

After the substitution of $\eta_{\mathrm{e}}=\eta_{\text {mag }}$ into the first of these 

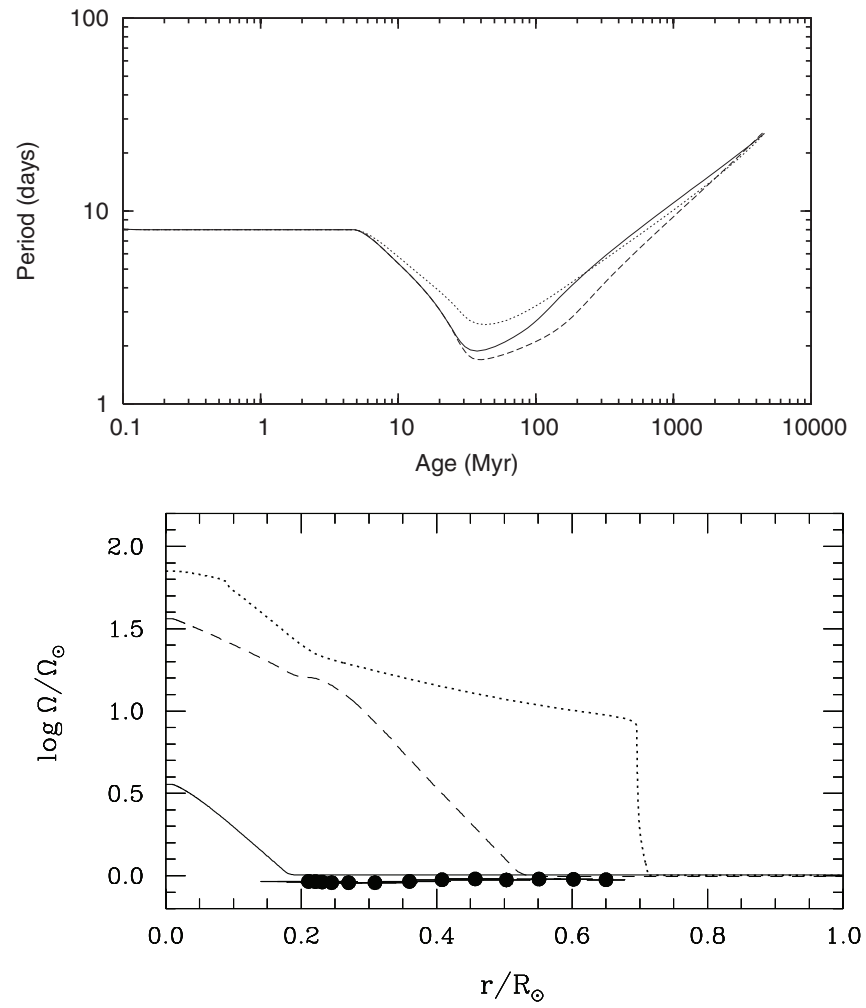

Figure 6. Rotation period evolution (upper panel) and final rotation profiles in the solar models (bottom panel) computed using Spruit's original (solid curves) and revised prescription (dashed curves). Dotted curves represent a model with no internal angular momentum transport. Filled circles in the bottom panel are helioseismic data from Couvidat et al. (2003).

equations, we find that the revised Tayler-Spruit dynamo ceases to work when the shear approaches a critical value

$$
q_{\min }=10^{2}\left(\frac{\varepsilon}{2+\varepsilon} \frac{N_{T}^{2}}{10^{-6}}+\frac{N_{\mu}^{2}}{10^{-6}}\right)^{1 / 2}\left(\frac{\Omega}{10^{-5}}\right)^{-1} .
$$

A comparison of coefficients in Equations (9) and (11) shows that in the second case the residual DR in the solar model is expected to be much stronger. A minimum value of $v_{\mathrm{e}}$ can be estimated from the second of Equations (10) in which we put $\eta_{\mathrm{e}}=\eta_{\mathrm{mag}}$ and $q=q_{\mathrm{min}}$. As a result, we obtain

$$
\begin{aligned}
\left(v_{\mathrm{e}}\right)_{\min }= & 1.69 \times 10^{6}\left(\frac{r}{R_{\odot}}\right)^{2 / 3}\left(\frac{\eta_{\mathrm{mag}}}{10^{3}}\right)^{2 / 3} \\
& \times\left(\frac{\varepsilon}{2+\varepsilon} \frac{N_{T}^{2}}{10^{-6}}+\frac{N_{\mu}^{2}}{10^{-6}}\right)^{-1 / 3}\left(\frac{\Omega}{10^{-5}}\right) \mathrm{cm}^{2} \mathrm{~s}^{-1},
\end{aligned}
$$

where all numbers are again expressed in cgs units.

It is instructive to compare the minimum values of the effective magnetic viscosity $\left(v_{\mathrm{e}}\right)_{\min }^{(8)}$ and $\left(v_{\mathrm{e}}\right)_{\min }^{(12)}$ given by Equations $(8)$ and (12), respectively. Their ratio is

$$
\begin{aligned}
\frac{\left(v_{\mathrm{e}}\right)_{\min }^{(8)}}{\left(\nu_{\mathrm{e}}\right)_{\min }^{(12)}}= & 41.2\left(\frac{r}{R_{\odot}}\right)^{1 / 3}\left(\frac{2+\varepsilon}{\varepsilon}\right)^{1 / 6}\left(\frac{\eta_{\mathrm{mag}}}{10^{3}}\right)^{-1 / 6} \\
& \times\left(\frac{N_{T}^{2}}{10^{-6}}\right)^{-1 / 6}\left(\frac{\Omega}{10^{-5}}\right)^{1 / 2} .
\end{aligned}
$$

The last two equations show that, even though the revised value of $\left(v_{\mathrm{e}}\right)_{\min }$ is less than the original one, it is still large enough for us to employ the same computational method that we used to study the evolution of rotating solar-type stars with Spruit's original prescription.

In Figure 6, results of our application of Spruit's original and revised prescriptions in full evolutionary computations of rotating solar models are compared with each other as well as with a rotating model with no internal transport of angular momentum (solid, dashed, and dotted curve, respectively). We confirm the conclusion made by Denissenkov \& Pinsonneault (2007), who used a crude approximation

$$
\Omega(r)=\Omega_{\odot}+\int_{r}^{r_{\text {bce }}} \Omega q_{\min } \frac{d r}{r}
$$

to construct an $\Omega$-profile in their model of the present-day Sun, that the revised prescription for the Tayler-Spruit dynamo produces a solar model with a large rapidly rotating core, in contradiction with helioseismic data (compare the dashed curve with the filled circles representing observational data from Couvidat et al. 2003 in bottom panel). Our new result obtained here is that, in spite of this, the revised prescription leads to the rotational evolution that has even shorter periods at ages older than $\sim 30$ Myr than the SB rotational evolution obtained with Spruit's original prescription (compare the dashed and solid curves in top panel). This behavior is explained as follows. As we anticipated, the values of $\left(v_{\mathrm{e}}\right)_{\min }$ given by Equation (12) indeed turned out to be large enough for the transport of angular momentum from the core to envelope to occur on a short "SB rotation" timescale like those we got in our constant viscosity models with $v_{0}>\left(v_{0}\right)_{\max } \approx 10^{6} \mathrm{~cm}^{2} \mathrm{~s}^{-1}$. However, because of an early build-up of a steep critical $\Omega$-profile (like that shown with dashed curve in bottom panel), below which the Tayler-Spruit dynamo ceases to work, the amount of the core's angular momentum available for transport to the envelope is diminished more and more as the evolution proceeds beyond an age of $\sim 30 \mathrm{Myr}$. As a result, if we used the value of the stellar wind parameter $K_{\mathrm{w}}=2.8 \times 10^{47} \mathrm{~cm}^{2} \mathrm{~g}$ s calibrated for Spruit's original prescription then the $P$-age relation for the revised prescription would be nearly parallel to the solid curve in top panel but it would be located at longer periods, hence the solar rotation period would be overestimated. To match the solar surface rotation, we had to reduce $K_{\mathrm{w}}$ to $1.25 \times 10^{47} \mathrm{~cm}^{2} \mathrm{~g} \mathrm{~s}$ for the revised prescription. This has shifted the $P$-age relation toward its location shown with the dashed curve. Note that we have also done test computations in which $\eta_{\mathrm{e}}$ from the first of Equations (10) was used instead of $\eta_{\text {mag }}$ to estimate $v_{\mathrm{e}}$. We have not found noticeable differences with the results obtained using the approximate method.

\subsection{Double-zone Model}

The two zone model was originally proposed by MacGregor (1991) and it has since been employed by many others (e.g., MacGregor \& Brenner 1991; Keppens et al. 1995; Siess \& Livio 1997; Allain 1998; Irwin et al. 2007). Angular momentum transport between the radiative core and convective envelope is parameterized as follows. The core and envelope, with moments of inertia and rotation rates $I_{\mathrm{c}}, \Omega_{\mathrm{c}}, I_{\mathrm{e}}$, and $\Omega_{\mathrm{e}}$ respectively, are assumed to rotate as solid bodies. If $\Omega_{\mathrm{c}}>\Omega_{\mathrm{e}}$ then the maximum angular momentum that can be transferred from the core to the envelope $(\Delta J)_{\max }$ is estimated as a difference between the core's initial angular momentum $J_{\mathrm{c}}=I_{\mathrm{c}} \Omega_{\mathrm{c}}$ and 
the angular momentum $J_{\mathrm{c}, \mathrm{eq}}=I_{\mathrm{c}} \Omega_{\mathrm{eq}}$ the core will have when its angular velocity becomes equal to that of the envelope. This final equilibrium velocity $\Omega_{\mathrm{eq}}$ is determined from the angular momentum conservation: $J_{\mathrm{c}}+J_{\mathrm{e}}=J_{\mathrm{c} \text {,eq }}+J_{\mathrm{e}, \mathrm{eq}}$, or $I_{\mathrm{c}} \Omega_{\mathrm{c}}+I_{\mathrm{e}} \Omega_{\mathrm{e}}=\left(I_{\mathrm{c}}+I_{\mathrm{e}}\right) \Omega_{\mathrm{eq}}$. So, we have

$$
(\Delta J)_{\max }=\frac{I_{\mathrm{c}} I_{\mathrm{e}}}{I_{\mathrm{c}}+I_{\mathrm{e}}}\left(\Omega_{\mathrm{c}}-\Omega_{\mathrm{e}}\right) .
$$

As a free parameter, the double-zone model uses the core/ envelope coupling time $\tau_{\mathrm{c}}$ that defines the rate $(\Delta J)_{\max } / \tau_{\mathrm{c}}$ with which the angular momentum is transferred from the core to the envelope. An advantage of this model is that one needs to know only how $I_{\mathrm{c}}, I_{\mathrm{e}}, r_{\mathrm{bce}}$, and $M_{\mathrm{bce}}$ are changing with time for a particular star (the last two quantities are required to take into account a displacement of the bottom of convective envelope during the angular momentum transfer). If this information is available (from full evolutionary computations) then the star's rotational evolution is obtained as a solution of the following system of ordinary differential equations (ODEs):

$$
\begin{gathered}
\frac{d J_{\mathrm{c}}}{d t}=-\frac{(\Delta J)_{\max }}{\tau_{\mathrm{c}}}+\frac{2}{3} r_{\mathrm{bce}}^{2} \Omega_{\mathrm{e}} \dot{M}_{\mathrm{bce}}, \\
\frac{d J_{\mathrm{e}}}{d t}=\frac{(\Delta J)_{\max }}{\tau_{\mathrm{c}}}-\frac{2}{3} r_{\mathrm{bce}}^{2} \Omega_{\mathrm{e}} \dot{M}_{\mathrm{bce}}+\dot{J}_{\mathrm{tot}},
\end{gathered}
$$

where $\dot{J}_{\text {tot }}$ should be replaced by expression (3).

Because of its simplicity the double-zone model can be used for Monte-Carlo simulations and other statistical studies, like those conducted in next section. However, before doing this we want to relate the constant viscosity model and the doublezone model to one another through their parameters $v_{0}$ and $\tau_{\mathrm{c}}$. In Figure 7, values of the coupling time are adjusted so that the double-zone model simulates some of the $P$-age relations that we computed with the constant viscosity model using the same initial conditions. We have established the following approximate ${ }^{3}$ correspondence between $\nu_{0}$ (in $\mathrm{cm}^{2} \mathrm{~s}^{-1}$ ) and $\tau_{\mathrm{c}}$ (in Myr): $\left(\nu_{0}, \tau_{\mathrm{c}}\right)=\left(2.5 \times 10^{4}, 90\right),\left(5 \times 10^{4}, 40\right),\left(10^{5}, 20\right)$, $\left(2.5 \times 10^{5}, 7\right)$, and $\left(10^{6}, 1\right)$. This means that the double-zone models with the coupling time longer than $\sim 40 \mathrm{Myr}$ correspond to the constant viscosity models that, by the solar age, still possess residual DR inconsistent with helioseismic data. On the other hand, the double-zone models with $\tau_{\mathrm{c}} \lesssim 1 \mathrm{Myr}$ reproduce the $P$-age relations for SB rotators (cf. Allain 1998); they are equivalent to the constant viscosity models with $\nu_{0}>$ $\left(v_{0}\right)_{\max } \approx 10^{6} \mathrm{~cm}^{2} \mathrm{~s}^{-1}$ as well as to the models in which angular momentum is redistributed by magnetic torques generated by the Tayler-Spruit dynamo in its original prescription.

\section{COMPARISON WITH OBSERVATIONS}

From a theoretical standpoint, the rotational evolution of lowmass stars is a very complex process. It depends on a number of parameters, such as the initial rotation period $P_{0}$, disk-locking time $\tau_{\mathrm{d}}$, angular velocity threshold for the magnetized wind saturation $\Omega_{\text {sat }}$, and the rate of angular momentum redistribution expressed in terms of the core/envelope coupling time $\tau_{\mathrm{c}}$, constant viscosity $\nu_{0}$, or as a function of stellar structure and other parameters when a particular physical mechanism is chosen to describe it. Because of this complexity, the progress

\footnotetext{
3 As the morphology of $P$-age relations is slightly different for these models, the adjusted values of $\tau_{\mathrm{c}}$ are uncertain within $\sim 10 \%$.
}
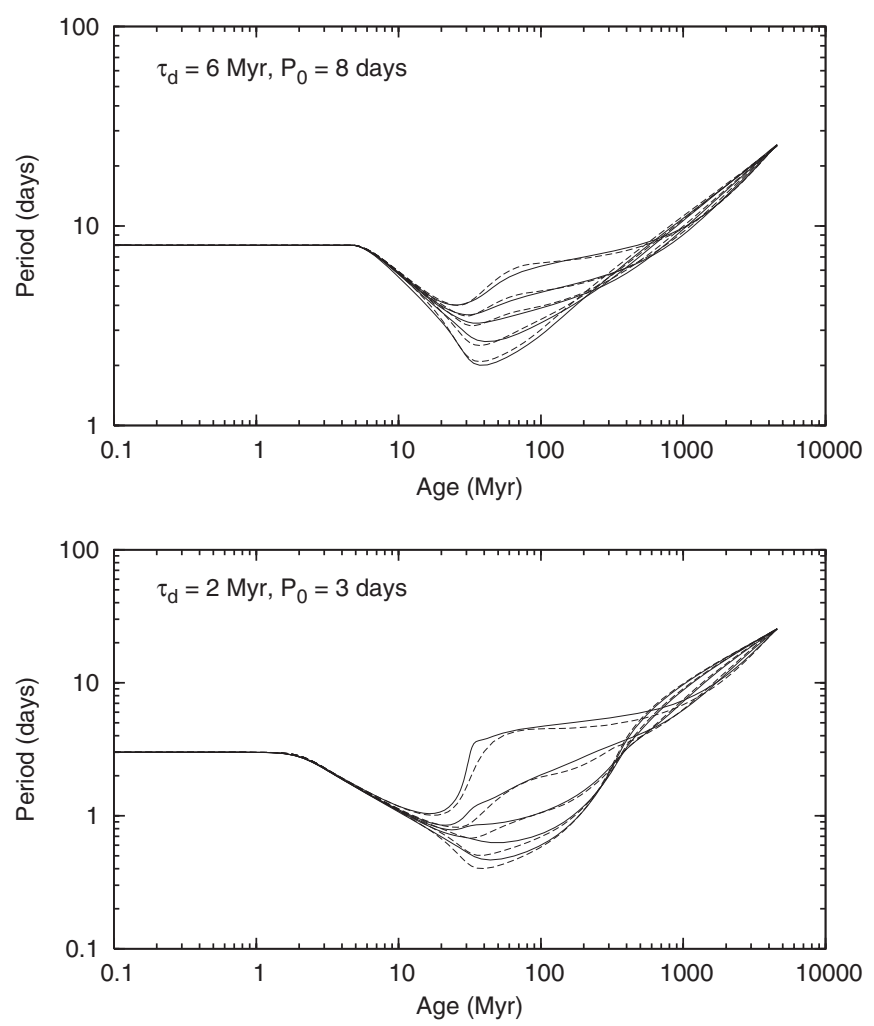

Figure 7. Comparison of rotation period evolution of the Sun computed using the constant viscosity (solid curves) and double-zone model (dashed curves). The corresponding pairs of the viscosity and core/envelope coupling time adjusted for the models' $P$-age relations to resemble one another as closely as possible are $\left(\nu_{0}, \tau_{\mathrm{c}}\right)=\left(2.5 \times 10^{4}, 90\right),\left(5 \times 10^{4}, 40\right),\left(10^{5}, 20\right),\left(2.5 \times 10^{5}, 7\right)$, and $\left(10^{6}, 1\right)$, where $v_{0}$ is given in $\mathrm{cm}^{2} \mathrm{~s}^{-1}$, and $\tau_{\mathrm{c}}$ in Myr.

in this field is primarily driven by constantly accumulating and improving observational data and their statistical analyses.

The basic method involves treating the open clusters as an evolutionary sequence: namely, that the distribution of initial conditions which produces the range of rotation rates seen in young clusters is a product of the star formation process and is independent of environment. Changes in the rotation distribution with age therefore reflect structural evolution, angular momentum loss from magnetized winds, and internal angular momentum transport. Weber \& Davis (1967) predicted an angular momentum loss rate which scales as $\Omega_{\mathrm{e}}^{3}$; this was confirmed by Skumanich (1972) and is well supported by studies of the asymptotic spin-down of old MS stars. Without a saturation in the effective loss rate, it would not be possible for rapid rotation to survive in young cluster stars (Pinsonneault et al. 1990; MacGregor \& Brenner 1991). A similar saturation in coronal $\mathrm{X}$-ray activity and chromospheric activity is also seen (Krishnamurthi et al. 1998). The properties of the upper envelope of the distribution are used to constrain the saturation threshold for rapid rotation.

The young slow rotators, however, are in the solar wind regime. As a result, their response to this milder applied torque can be used to diagnose the timescale over which the core couples to the envelope. In the limit of weak coupling, the envelope spins down quickly because of its small moment of inertia; this stalls when the flow of angular momentum from the core balances the applied torque. Surface spin-down resumes at older ages when the core and envelope are coupled (see Pinsonneault et al. 1989). By contrast, in the limit of SB rotation at all times, measurable spin-down should not be seen in young 
clusters and there is no physical mechanism which produces rapid early spin-down, followed by a pause and a resumption of spin-down at later ages. The fraction of the moment of inertia in the surface convection zone is a strong function of mass, so the method can also be tested by comparing the spin-down of nearly fully convective stars (where the moment of inertia of the core is too small to impact spin-down) to stars with thinner surface convection zones.

A number of physical mechanisms have been proposed to explain the solar SB rotation, such as the smoothing of DR by the back reaction of the Lorentz force emerging from the generation of a toroidal magnetic field by shearing of a preexisting poloidal field (Mestel \& Weiss 1987; Charbonneau \& MacGregor 1993; Denissenkov 2010), angular momentum redistribution by magnetic torques generated by the Tayler-Spruit dynamo (Eggenberger et al. 2005), or by internal gravity waves excited by turbulent eddies in the solar convective envelope (Charbonnel \& Talon 2005; see, however, Denissenkov et al. 2008). However, none of these mechanisms have been shown to agree or disagree with available rotation period data for solar-type stars in open clusters. In this paper, we subject the Tayler-Spruit dynamo to such an observational test. The other mechanisms will be tested in our forthcoming papers. We will start with Spruit's original prescription. As it always results in the $P$-age relations similar to those obtained with the double-zone model having a short coupling time of order 1 Myr (compare Figures 5 and 7), we will use the latter as its substitute model. The main advantage of this replacement is that the double-zone model computations are very fast, therefore they can effectively be used to perform extensive parameter-space investigations.

Our first step in reducing the number of free model parameters is the adjustment of the value of $\Omega_{\text {sat }}$ (see Section 2.3). For the SB rotating $\left(\tau_{\mathrm{c}}=1 \mathrm{Myr}\right)$ double-zone model to reproduce as close as possible the upper 90th percentiles simultaneously for all of our compiled $\Omega_{\mathrm{e}}$ data for each of the three mass bins, we had to choose $\Omega_{\text {sat }}=8 \Omega_{\odot}$ for our two "solar mass" bins and $\Omega_{\text {sat }}=2.5 \Omega_{\odot}$ for the "fully convective" mass bin (upper solid curves in Figure 3, these curves are also plotted in Figure 1 , with $\Omega_{\mathrm{e}}$ being transformed back to $P$ ). This procedure assumes quite naturally that the most rapidly rotating stars in the samples evolve as SB rotators. In fact, it is impossible to construct a double-zone model with DR that would fit the 90th percentiles for the intermediate-age $(\sim 100 \mathrm{Myr}$ old $)$ clusters, those located immediately behind the maxima on the $\Omega_{\mathrm{e}}$ versus age curves, without making unreasonable assumptions about its parameters.

It turns out that the lower 10th percentiles for the stars with $M \leqslant 0.4 M_{\odot}$ can also be approximated reasonably well with an SB rotation evolution curve (the lower and upper solid curves in Figures 3(c) and 1(c), respectively). This result is expected because a turbulent eddy viscosity in these fully convective stars should redistribute angular momentum very quickly (e.g., see Tinker et al. 2002). Having said that, we should mention a recent evidence that casts some doubt on this simple interpretation. The first period measurements for five stars with masses below $0.5 M_{\odot}$ in Praesepe (e.g., Scholz \& Eislöffel 2007) appear to disagree with the predicted model trends, but this sample is not of sufficient size to draw firm conclusions.

In contrast, it turns out to be very difficult for an SB rotation evolution curve to approach all the lower 10th percentiles for stars in the mass bins centered at $1.0 M_{\odot}$ and $0.8 M_{\odot}$ (dashed curves in panels (a) and (b) in Figure 3), unless one takes a disk-locking time well in excess of 20 Myr or starts with a very slowly rotating star. If the double-zone model parameters were not constrained by observational data but could be chosen at our will then any mechanism of angular momentum transport that persistently produces and maintains SB rotation, in particular Spruit's original prescription, could easily be brought in agreement with the open cluster rotation period data for slowly rotating solar-type stars. Indeed, in this case one could simply choose appropriate combinations of the initial period and disklocking time, one or both of which having to be sufficiently long, such that the double-zone model with SB rotation $\left(\tau_{\mathrm{c}}=1 \mathrm{Myr}\right)$ and with those parameters applied would embrace all the periods for slow rotators, no matter how long they are (dashed and dotdashed curves in panels (a) and (b) in Figure 1).

However, observations do not allow such an arbitrary choice of parameters of a rotational evolution model for solar-type stars. In particular, the typical disk-locking time has been estimated to lie in a range between 2 and $10 \mathrm{Myr}$. This result is based on the measuring of such diagnostics of the presence of a circumstellar disk around a pre-MS star as an IR excess, that traces dust, or an $\mathrm{H}_{\alpha}$ emission line width that traces accretion (Hillenbrand 2005; Lyo \& Lawson 2005; Bouwman et al. 2006; Jayawardhana et al. 2006; Damjanov et al. 2007). A recent statistical analysis of available data on the pre-MS circumstellar disks has led Mamajek (2009) to the conclusion that "the fraction of young stars with optically thick primordial disks and/or those which show spectroscopic evidence for accretion appears to approximately follow an exponential decay with characteristic time 2.5 Myr." Taken at its face value, this means that, on average, only $10 \%$ of active disks around pre-MS solar-type stars are expected to survive by the age of 5.8 Myr. However, the dispersion of the disk life times can be quite large. For instance, Sicilia-Aguilar et al. (2009) have found that $\sim 50 \%$ of 18 members of the $\eta$ Cham cluster studied by them still show signatures of a circumstellar disk by the age of 8 Myr. Therefore, in our models we will use the maximum disk-locking time $\left(\tau_{\mathrm{d}}\right)_{\max }=20$ Myr as a safe upper limit.

With the maximum disk-locking time limited by the value of $10 \mathrm{Myr}$ or even $20 \mathrm{Myr}$, the SB rotational evolution can explain the longest periods in the intermediate-age clusters only if it starts with initial periods that are much longer than those measured in the youngest clusters (dashed and dotdashed curves in panels (a) and (b) in Figure 1). However, the assumption that individual open clusters had their unique distributions of $P_{0}$ in the past, with very different statistics, does not seem realistic because the same laws of physics had most likely shaped them during the star formation. This conclusion is supported by the fact that the period distributions for solartype stars in three of our youngest clusters, ONC, NGC 2264, and NGC 2362, have high KS probabilities of having been drawn from the same real distribution (Figure 2). Therefore, we will assume that the period distributions of stars in clusters of different ages $f(t, P)$ represent an evolutionary sequence that started with the same distribution $f\left(0, P_{0}\right)$. Besides, we will consider the initial distribution of disk-locking times to be flat and random in the interval $0<\tau_{\mathrm{d}} \leqslant\left(\tau_{\mathrm{d}}\right)_{\max }$ with the maximum value of $\left(\tau_{\mathrm{d}}\right)_{\max }=20 \mathrm{Myr}$. As a proxy for $f\left(0, P_{0}\right)$, we will take period distributions for the three aforementioned young clusters. If the initial rotation of cluster stars was much slower than predicted by the ONC data then we should see clusters arriving on the MS with very low rotation rates, e.g., systems with ages below $100 \mathrm{Myr}$ should already have a lot of slow rotators. Small samples from very young open clusters exhibit no such trend, but larger sample sizes are required. 

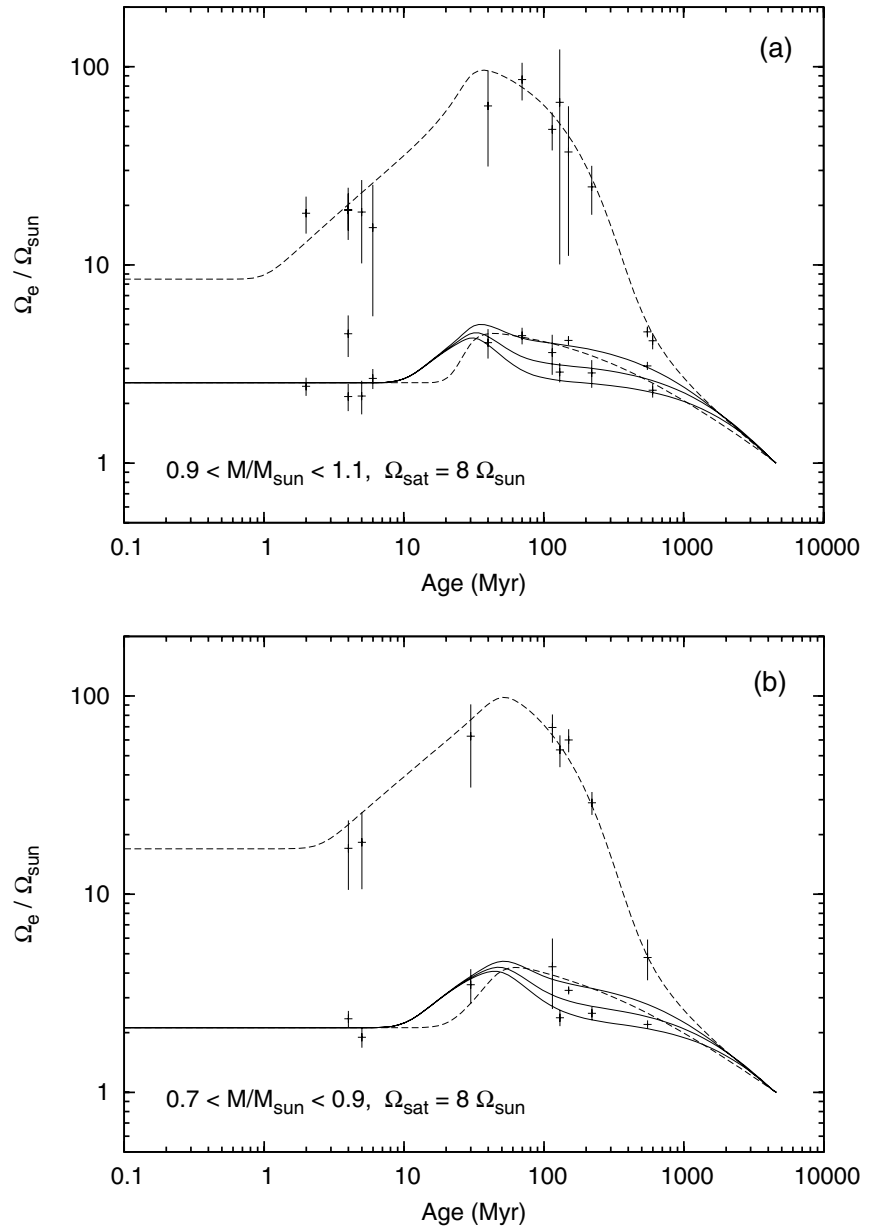

Figure 8. Upper 90th and lower 10th percentiles (crosses) of $\Omega_{\mathrm{e}}$ distributions for all of our compiled data samples of open cluster solar-type stars are compared with $\Omega_{\mathrm{e}}$-age relations computed using the double-zone model. Dashed curves correspond to $\tau_{\mathrm{c}}=1 \mathrm{Myr}$ (SB rotation), solid curves (from upper to lower) to DR with $\tau_{\mathrm{c}}=50,100$, and $150 \mathrm{Myr}$ (panel (a)), and $\tau_{\mathrm{c}}=100,200$, and $300 \mathrm{Myr}($ panel (b))

The assumptions that we have just made are not novel. For instance, Irwin et al. (2007) evolved the observed rotation rates of low-mass stars in NGC 2362 forward in time trying to reproduce some statistics of $\Omega_{\mathrm{e}}$ distributions in older clusters. Like us, they employed a double-zone model. To compare the results of their computations with observations, Irwin et al. (2007) calculated the lower 25th and upper 90th percentiles of the observed distributions of $\Omega_{\mathrm{e}}$ for solar-type stars in NGC 2362, IC 2391/IC 2602, $\alpha$ Per, M 34, and the Hyades. These statistics have been chosen to characterize the slowest and fastest rotators in the selected clusters. Using our doublezone model with $\tau_{\mathrm{c}}=1 \mathrm{Myr}$, we confirm the conclusion made by Irwin et al. (2007) that the rotational evolution of the fastest rotators among solar-type stars can be simulated closely enough with short coupling times characteristic of SB rotators (upper dashed curves in Figure 8). In contrast, much longer coupling times of order $\tau_{\mathrm{c}}=50-150 \mathrm{Myr}$ and $\tau_{\mathrm{c}}=100-300 \mathrm{Myr}$ for the mass bins $0.9 \leqslant M / M_{\odot} \leqslant 1.1$ and $0.7 \leqslant M / M_{\odot}<0.9$, respectively, as obtained in our computations (solid curves in the same figure), are required to explain the rotational evolution of the slowest rotators. ${ }^{4}$

\footnotetext{
4 Irwin et al. (2007) reported much longer coupling times because they did not recalibrate the wind parameter $K_{\mathrm{w}}$ for the slowest rotators.
}

From a theoretical standpoint, the inability of the SB rotation model, on the one hand, and the ability of the DR model, on the other hand, to reproduce the location of the slowest rotators in the $\Omega_{\mathrm{e}}$-age plot is caused by their, respectively, inappropriate and appropriate characteristics of mapping of $f\left(0, P_{0}\right)$ to $f(t, P)$ for long rotation periods. Apparently, the still unknown physical mechanism responsible for the internal angular momentum transport in solar-type stars must have a property, which is most likely related to $\Omega_{\mathrm{e}}$, of making rotation of the radiative core and convective envelope more and more decoupled as one goes from a faster to slower rotating star. For example, the timescale for internal angular momentum transport by hydrodynamic mechanisms becomes shorter as the rotation rate increases in this fashion (Pinsonneault et al. 1990). Assuming that a quantitative characteristic describing this property changes continuously with $\Omega_{\mathrm{e}}$ (or $P$ ), this should result in qualitative differences between the true $\Omega_{\mathrm{e}}$ distributions for evolved clusters and those obtained with the double-zone model for different values of $\tau_{\mathrm{c}}$. These qualitative differences can be put on a scale by comparing the observed distributions of $\Omega_{\mathrm{e}}$ with the modeled ones using the KS test. This procedure is more rigorous than just matching the percentiles, because it uses statistical information encoded in the entire distribution rather than only in a part of it.

Given the aforementioned robust results concerning the fastest solar-type rotators, the basic assumption of our following statistical analysis is that the rotational evolution of stars with sufficiently short initial rotation periods can be described by the double-zone model with $\tau_{\mathrm{c}}=1 \mathrm{Myr}$, whereas that of slower rotating stars needs $\tau_{\mathrm{c}} \gg 1 \mathrm{Myr}$. In principle, we ought to introduce some monotonically increasing function $\tau_{\mathrm{c}}\left(P_{0}\right)$ into our double-zone model that would produce a smooth transition from short-period SB rotators to stars with progressing DR that had longer initial periods. However, given the simplicity of the double-zone model, such approach looks overcomplicated. Therefore, we have decided to employ a simple step function

$$
\tau_{\mathrm{c}}\left(P_{0}\right)= \begin{cases}1 \mathrm{Myr}, & \text { if } P_{0} \leqslant P_{\mathrm{c}}, \\ \tau_{\mathrm{c}} \gg 1 \mathrm{Myr}, & \text { if } P_{0}>P_{\mathrm{c}},\end{cases}
$$

where $P_{\mathrm{c}}$ is a critical period.

The main objective of our statistical analysis of open cluster rotation period data for solar-type stars is to get estimates of the combination of parameters $\left(P_{\mathrm{c}}, \tau_{\mathrm{c}}\right)$ from Equation (16) that give the highest KS probabilities of the hypothesis that an observed $\Omega_{\mathrm{e}}$ distribution for an open cluster of age $t$ and our theoretical $\Omega_{\mathrm{e}}$ distribution computed for the same age $t$ have been drawn from the same real distribution. It is assumed that the initial period distribution $f\left(0, P_{0}\right)$ is provided by the youngest clusters from our data compilation: ONC, NGC 2362, or NGC 2264 (Table 1). To do the analysis, we have performed extensive double-zone model computations densely covering relevant regions (squares) of the $\left(\left(\tau_{\mathrm{d}}\right)_{\max }, \tau_{\mathrm{c}}\right)$ parameter space for a number of $P_{\mathrm{c}}$ values. The resulting $P_{\mathrm{KS}}$ contours are plotted in Figure 9 (the mapping of ONC to M 35 for the mass bin centered at $M=1.0 M_{\odot}$ ), Figure 10 (NGC 2362 to $\mathrm{M} 50$ for $M=1.0 M_{\odot}$ ), Figure 11 (the mapping of NGC 2362 to M 34 for the mass bin centered at $M=0.8 M_{\odot}$ ), and Figure 12 (NGC 2362 to $\mathrm{M} 50$ for $\left.M=0.8 M_{\odot}\right)$.

The $P_{\mathrm{KS}}$ contour patterns revealed in Figures 9-11 clearly show a decrease of the most probable value of the coupling time with an increase of the disk-locking time, as expected. Indeed, 

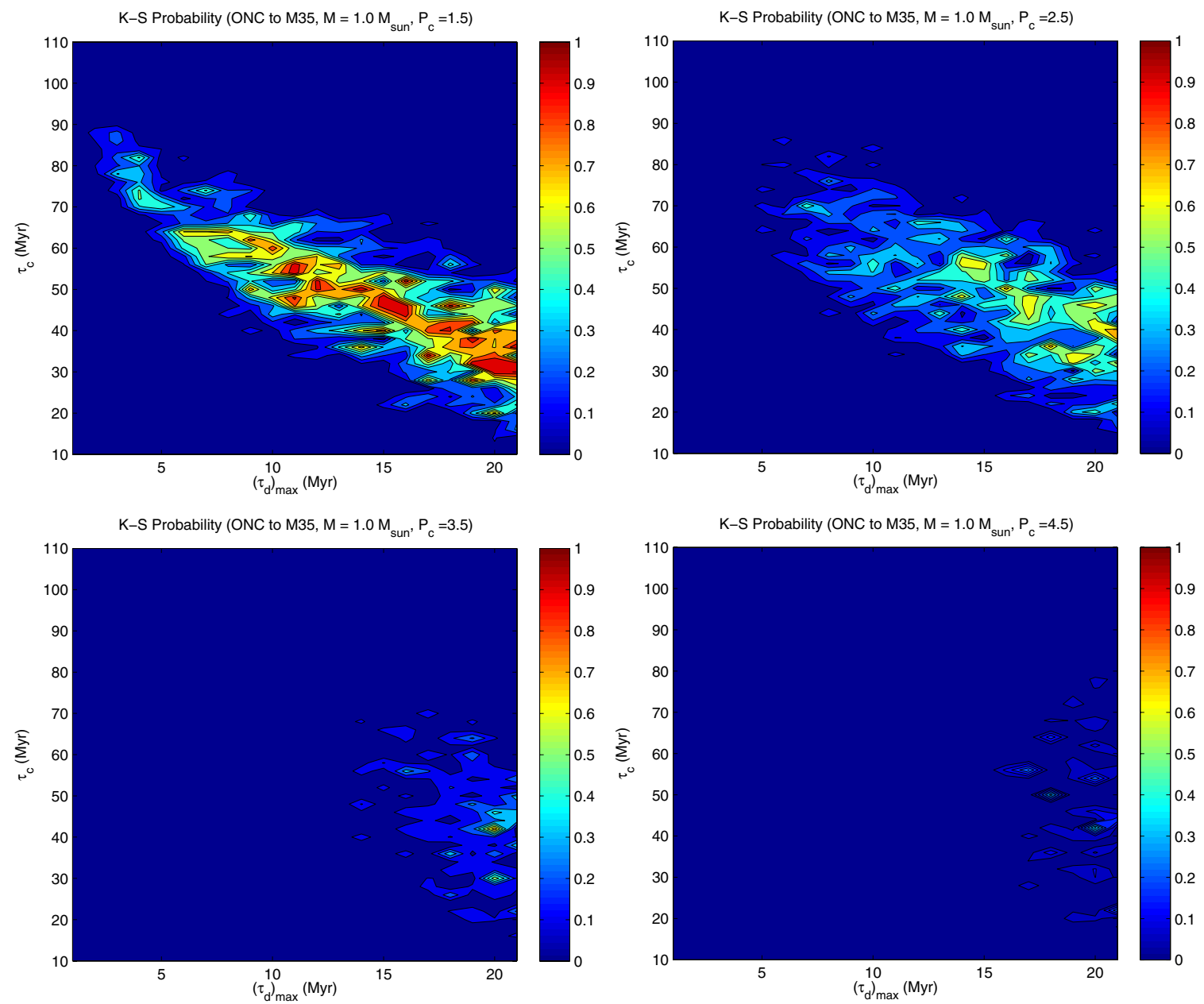

Figure 9. Contours of the Kolmogorov-Smirnov probability of the hypothesis that the observed and modeled period distributions of M 35 stars with $M=1.0 \pm 0.1 M M_{\odot}$ have been drawn from the same real distribution. The initial periods are taken from the ONC cluster. The double-zone model with the dependence (16) of $\tau_{\mathrm{c}}$ on $P_{0}$ has been employed. Simulations have been done for the shown intervals of the parameters $\tau_{\mathrm{c}}$ and $\left(\tau_{\mathrm{d}}\right)_{\max }$, and for the values of $P_{\mathrm{c}}$ specified in parenthesis atop each panel.

(A color version of this figure is available in the online journal.)

a star whose pre-MS rotation rate was being kept constant, as a result of its interaction with a circumstellar disk, for a longer time would arrive at the ZAMS having a smaller amount of angular momentum stored in the radiative core. Therefore, even a shorter coupling time will not lead to a fast rotation of its convective envelope simply because there is not much of angular momentum left in the core to be transported to the envelope.

From Figure 2 and Table 1, we can see that there is a large number of stars in the youngest clusters with periods $P_{0}<12$ days. Yet the $P_{\mathrm{KS}}$ contour patterns in Figures 9-11 first become apparent, as $P_{\mathrm{c}}$ increases, and then get dissolved well before the critical period reaches the value of 12 days. This means that the double-zone model cannot reproduce the $\Omega_{\mathrm{e}}$ distributions in evolved clusters under the assumption that all stars are SB rotators. There is always a group of stars with rotation of the radiative core decoupled from that of the convective envelope. For the mass bin centered at $1.0 M_{\odot}$, a lower limit for the coupling time can be estimated as $\tau_{\mathrm{c}} \gtrsim 30 \mathrm{Myr}$ for $\left(\tau_{\mathrm{d}}\right)_{\max }=20 \mathrm{Myr}$, and $\tau_{\mathrm{c}} \gtrsim 55 \mathrm{Myr}$ for $\left(\tau_{\mathrm{d}}\right)_{\max }=10 \mathrm{Myr}$ (Figure 9). Figure 10 gives nearly $20 \mathrm{Myr}$ longer coupling times. Probable values of the parameter $P_{\mathrm{c}}$ that divides stars into SB rotators and objects possessing DR range from 2.5 to 5.5 days for these two mappings. However, what is more important for us here is that, in all considered cases, we can completely rule out the pure SB rotation evolution $\left(P_{\mathrm{c}}=\infty\right)$ as a solution. Consequently, Spruit's mechanism or any other physical mechanism that can only produce nearly SB rotation should be rejected as a prescription for rotational evolution of solar-type stars. The true model should have a parameter (presumably related to $\Omega_{\mathrm{e}}$ ) that allows it to tune up its rotational evolution so that the latter would resemble the rotational evolution of the double-zone model with the coupling time changing from $\tau_{\mathrm{c}}=1 \mathrm{Myr}$ to $\tau_{\mathrm{c}} \approx 55 \mathrm{Myr}$. For stars with masses in the interval $0.7 \leqslant M / M_{\odot}<0.9$, we estimate much longer coupling times: $\tau_{\mathrm{c}} \gtrsim 150 \mathrm{Myr}$ for $\left(\tau_{\mathrm{d}}\right)_{\max }=20 \mathrm{Myr}$, and $\tau_{\mathrm{c}} \gtrsim 200 \mathrm{Myr}$ for $\left(\tau_{\mathrm{d}}\right)_{\max }=10 \mathrm{Myr}$ (Figures 11 and 12 ). This result can be anticipated from Figure 8 where we compare the lower 10th percentiles with our DR double-zone model computations.

As an additional statistical exercise aiming to demonstrate the failure of SB rotation to serve as a unique solution for all solartype stars, we have evolved the observed period distributions for M 35 and M 50 backward in time to zero age, assuming the short coupling time of $1 \mathrm{Myr}$ for all stars, to see how their initial period distributions might resemble those of Orion and NGC 2264, respectively. To solve this inverse problem, we have first projected (using our double-zone model with $\tau_{\mathrm{c}}=1 \mathrm{Myr}$ ) 

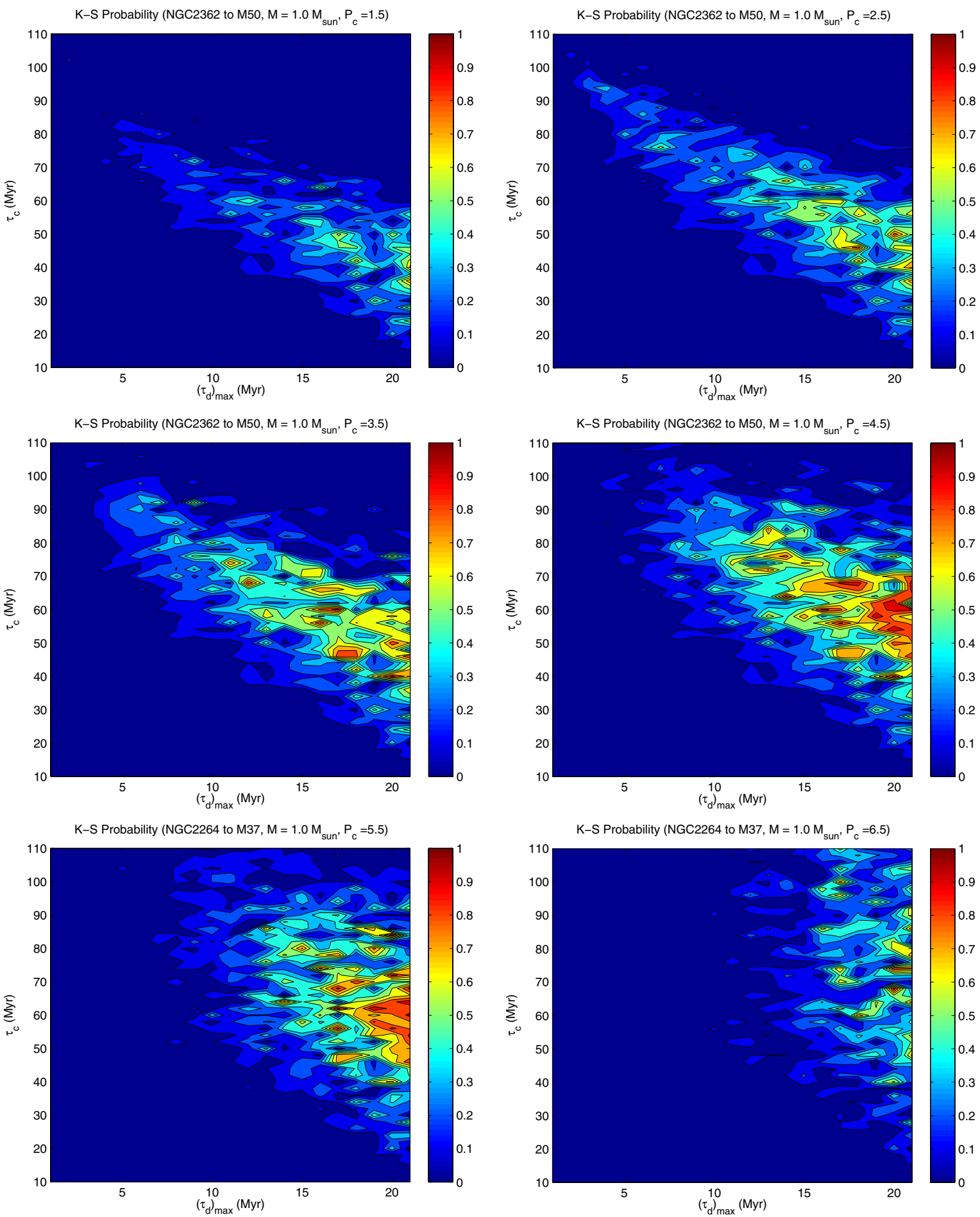

Figure 10. Contours of the Kolmogorov-Smirnov probability of the hypothesis that the observed and modeled period distributions of M 50 stars with $M=1.0 \pm 0.1 M_{\odot}$ have been drawn from the same real distribution. The initial periods are taken from the NGC 2362 cluster. The double-zone model with the dependence (16) of $\tau_{\mathrm{c}}$ on $P_{0}$ has been employed. Simulations have been done for the shown intervals of the parameters $\tau_{\mathrm{c}}$ and $\left(\tau_{\mathrm{d}}\right)_{\max }$, and for the values of $P_{\mathrm{c}}$ specified in parenthesis atop each panel.

(A color version of this figure is available in the online journal.)

a sufficiently wide rectangular domain of the $\left(\log P_{0}, \log \tau_{\mathrm{d}}\right)$ parameter space into the $\log P$ space at the ages of M 35 and M 50. The projections have turned out to look like perfect planes. Contour lines for the M 35 projection are plotted in upper panel in Figure 13. It is seen that the backward solution is not unique. In fact, every value of $\log P(t)$ at an older age $t$ is mapped into a diagonal line segment

$$
\log P_{0} \approx a \log \tau_{\mathrm{d}}+b
$$

back at $t=0$. To assign a unique value of the initial period for $\log P(t)$, we have decided to randomly select $\tau_{\mathrm{d}}$ from an interval 

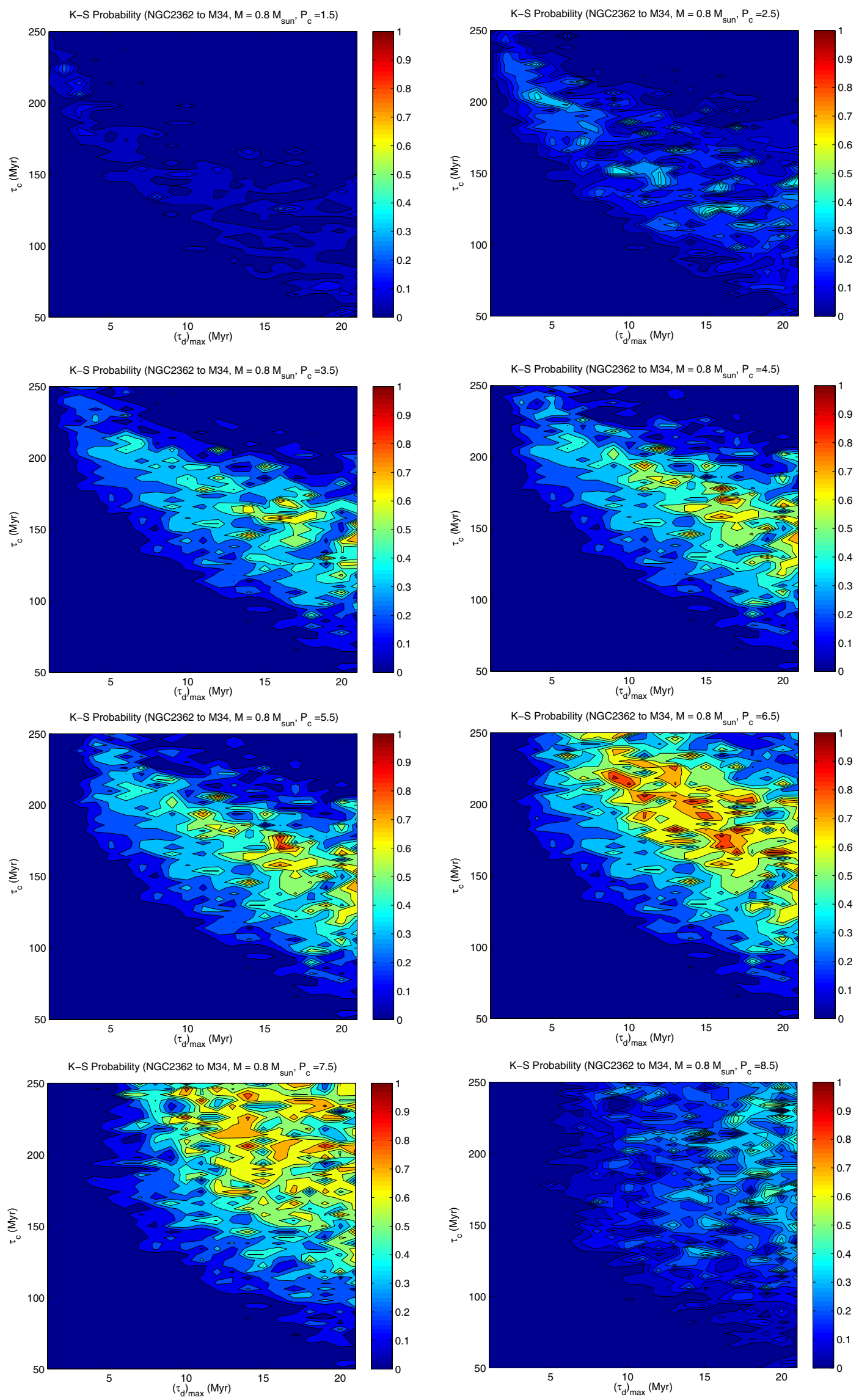

Figure 11. Contours of the Kolmogorov-Smirnov probability of the hypothesis that the observed and modeled period distributions of M 34 stars with $M=0.8 \pm 0.1 M_{\odot}$ have been drawn from the same real distribution. The initial periods are taken from the NGC 2362 cluster. The double-zone model with the dependence (16) of $\tau_{\mathrm{c}}$ on $P_{0}$ has been employed. Simulations have been done for the shown intervals of the parameters $\tau_{\mathrm{c}}$ and $\left(\tau_{\mathrm{d}}\right)_{\max }$, and for the values of $P_{\mathrm{c}}$ specified in parenthesis atop each panel.

(A color version of this figure is available in the online journal.) 
K-S Probability ( $N G C 2362$ to $M 50, M=0.8 M_{\text {sun }}, P_{c}=1.5$ )

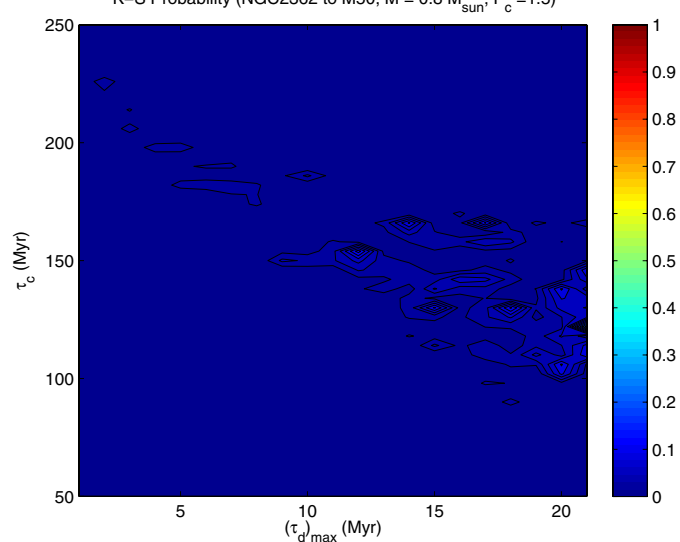

K-S Probability ( $N G C 2362$ to $M 50, M=0.8 M_{\text {sun, }}, P_{c}=3.5$ )

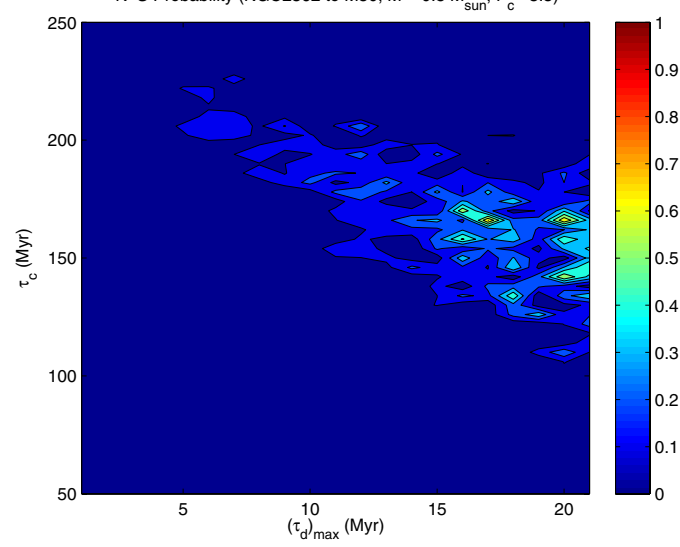

K-S Probability ( $N G C 2362$ to $M 50, M=0.8 M_{\text {sun }}, P_{c}=5.5$ )

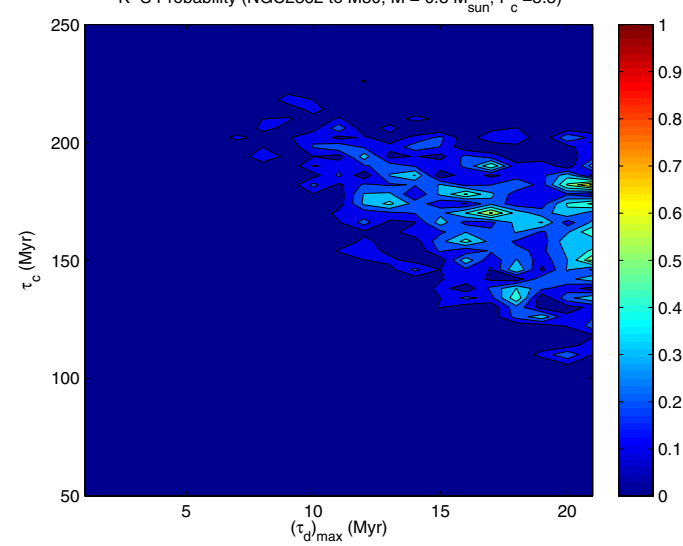

K-S Probability (NGC2362 to $M 50, M=0.8 M_{\text {sun, }}, P_{c}=7.5$ )

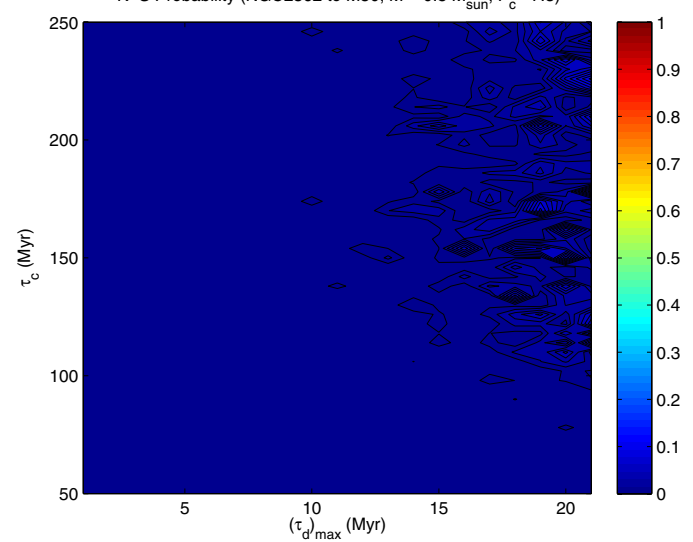

K-S Probability ( $N G C 2362$ to $M 50, M=0.8 M, P_{c}=2.5$ )

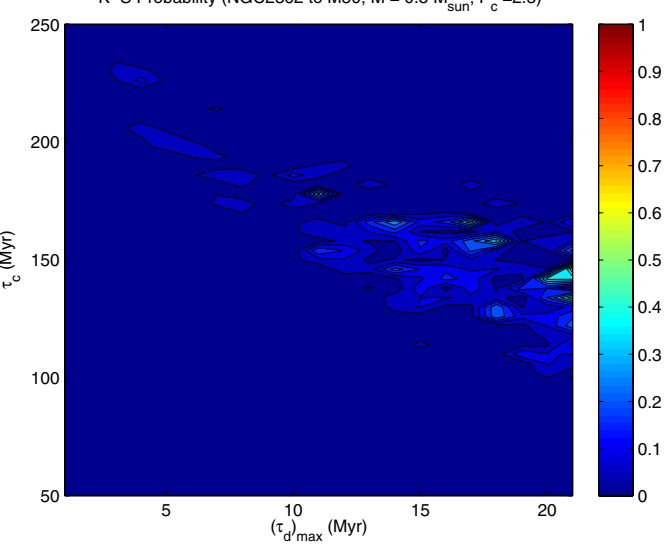

K-S Probability (NGC2362 to $M 50, M=0.8 M_{\text {sun }}, P_{c}=4.5$ )

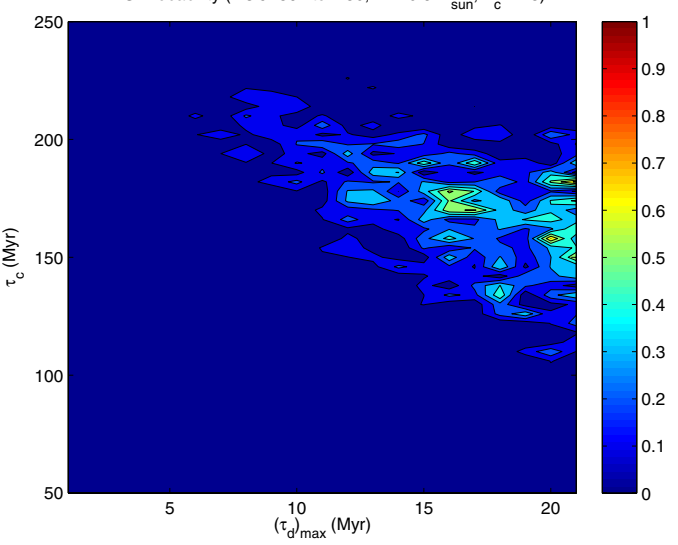

K-S Probability ( $N G C 2362$ to $M 50, M=0.8 M_{\text {sun }}, P_{c}=6.5$ )

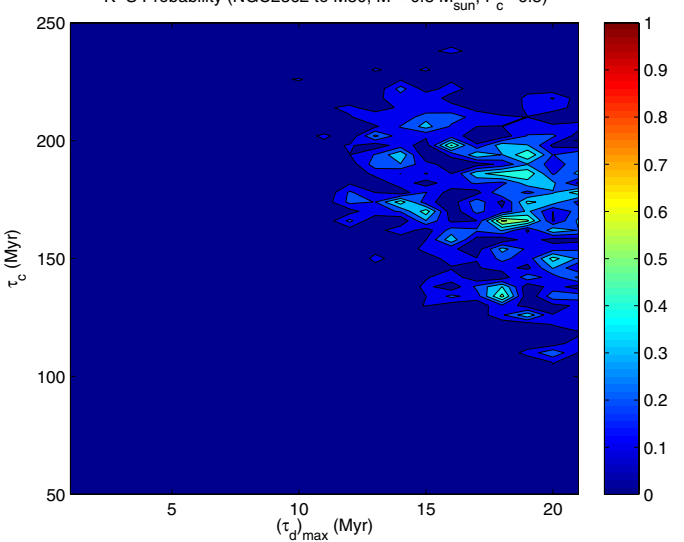

K-S Probability (NGC2362 to $M 50, M=0.8 M_{\text {sun }}, P_{c}=8.5$ )

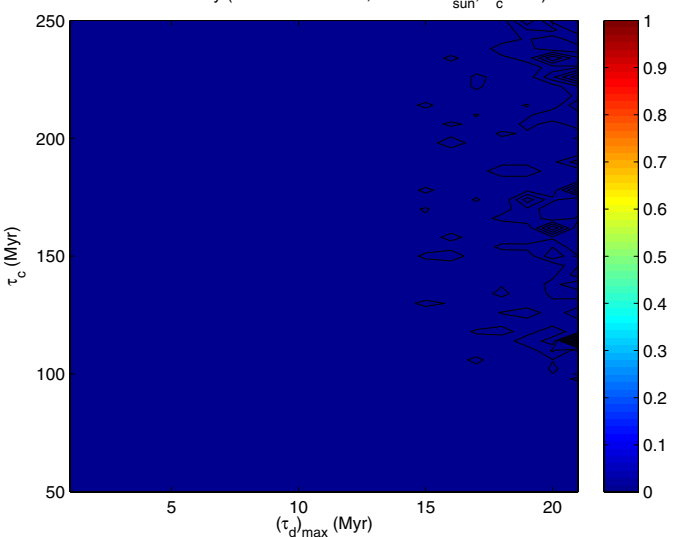

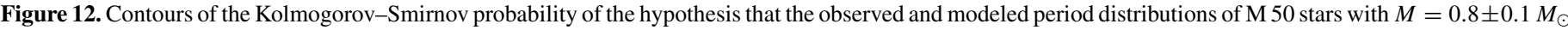

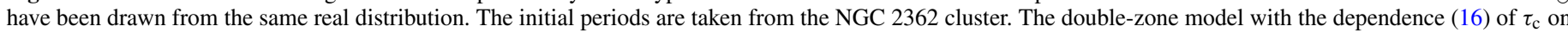

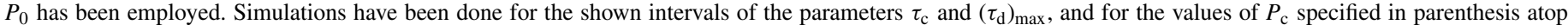
each panel.

(A color version of this figure is available in the online journal.) 

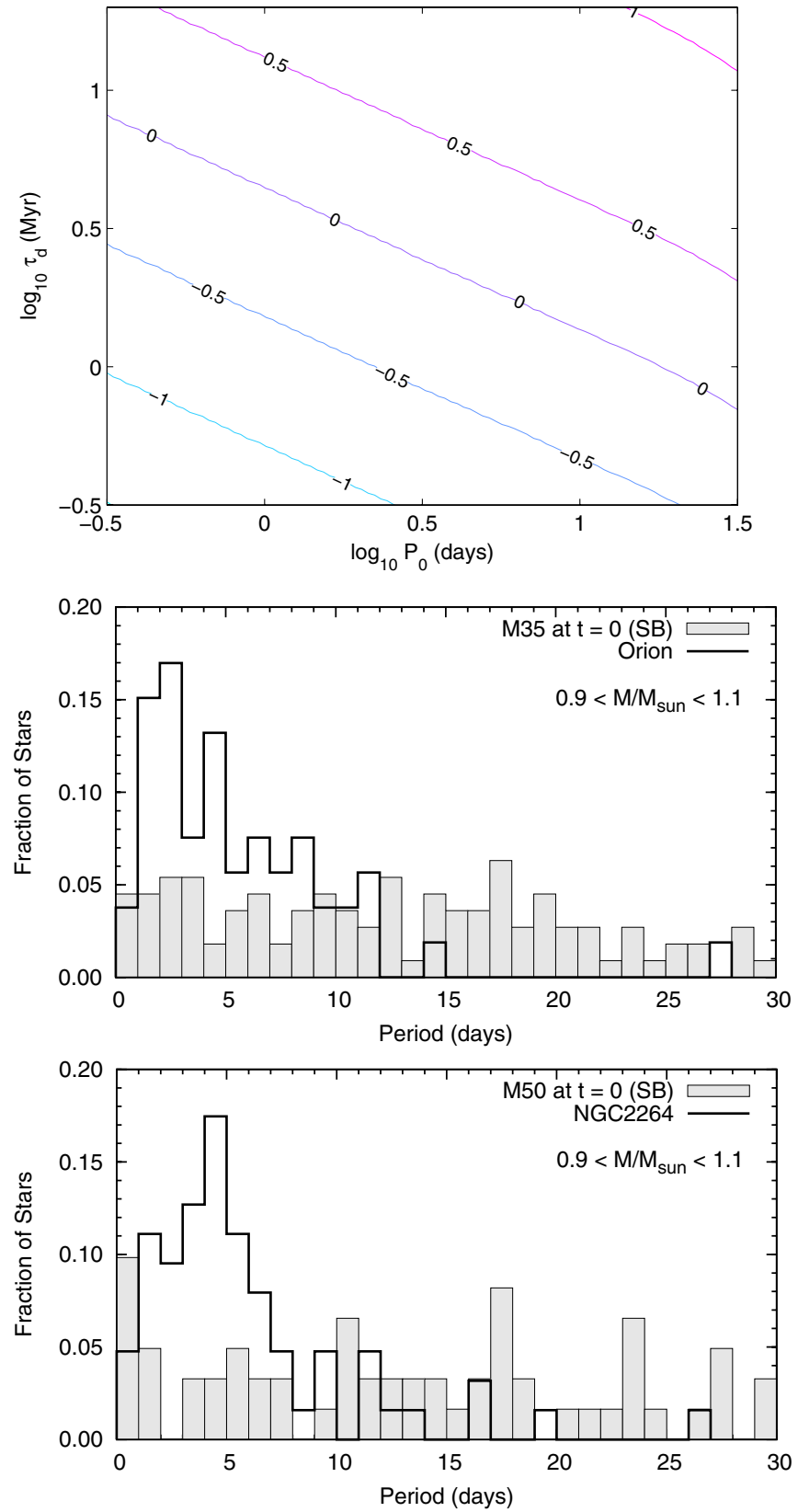

Figure 13. Upper panel: contour lines in a domain of initial conditions $\left(\log P_{0}, \log \tau_{\mathrm{d}}\right.$ ) that are projected to specified values of $\log P$ (shown on the lines) at the age of M 35 (150 Myr) for SB rotational evolution computed using the double-zone model with $\tau_{\mathrm{c}}=1 \mathrm{Myr}$. These lines are used to restore the initial period distribution for solar-type stars in M 35 (shaded histogram in middle panel) and to compare it with the Orion period distribution (thick solid curve). Bottom panel shows the corresponding comparison between M 50 and NGC 2264.

(A color version of this figure is available in the online journal.)

restricted by the end points of the diagonal (Equation (17)). Thus computed "initial" rotation periods for the M 35 and M 50 stars are compared with the Orion and NGC 2264 period distributions in Figure 13 (middle and bottom panels).

We see that the hypothesis that all solar-type stars, independently of their rotation periods, evolved like SB rotators would require that M 35 and M 50 had initially contained much larger fractions of slow rotators compared to Orion and NGC 2264. Because this is unlikely to be the case, we should reject any prescription for the internal transport of angular momentum that enforces SB rotation in a solar-type star without respect to how slowly it rotates. In particular, the original Tayler-Spruit dynamo cannot be considered as a relevant mechanism for angular momentum redistribution in solar-type stars because it always enforces nearly SB rotation in both fastest and slowest rotators.

\section{DISCUSSION AND CONCLUSIONS}

One of the main conclusions made in this paper is that the observed rotation period distributions of low-mass stars in open clusters do not seem to support the hypothesis that all solar-type stars evolve as SB rotators. Instead, statistical analyses of these data show that only the fastest rotators among solar-type stars can be considered to possess SB rotation during their entire evolution, whereas their slowly rotating counterparts are most likely to manifest internal DR between the ages of $\sim 30 \mathrm{Myr}$ and several hundred Myr. This is not a new result though because, e.g., Irwin et al. (2007) came to a similar conclusion. A novelty of our work is that we have used the entire period distributions of solar-type stars in a number of open clusters of different ages, not just some of their statistics, and extensive MonteCarlo simulations to put this conclusion on a more rigorous quantitative basis. This seems to be quite a reasonable approach to the solution of the problem, given that it has a large number of poorly constrained parameters. In particular, we have found that a star with $M=1.0 \pm 0.1 M_{\odot}$ that starts its rotational evolution with a period $P_{0} \gtrsim 2-4$ days should have the rotation of its convective envelope and radiative core coupled on a timescale of order $\tau_{\mathrm{c}}=55 \pm 25 \mathrm{Myr}$, where the systematic uncertainty of this estimate takes into account the anticipated decrease of the coupling time with an increase of the disk-locking time. For a slightly less massive star with $M=0.8 \pm 0.1 M_{\odot}$, the coupling time increases to $\tau_{\mathrm{c}}=175 \pm 25$ Myr. Given that the initial period distributions, those for the youngest open clusters, have rather densely occupied bins up to $P_{0} \approx 12$ days (Figure 2 ), it turns out that quite large fractions of solar-type stars (up to $50 \%$ ) should go through a phase of DR evolution.

It is important to note that solar-type stars in open clusters older than a few hundred Myr are not suitable for a type of statistical analysis employed by us. The problem with the older clusters is that rotation periods of solar-type stars in them have already converged too close to each other, all aiming eventually to approach the solar rotation. Therefore, period distributions for the older clusters do not allow to make an unambiguous conclusion about the rotational evolution of solar-type stars. To illustrate this, we have evolved the NGC 2264 period distribution of stars with $M=1.0 \pm 0.1 M_{\odot}$ to a period distribution at $t=550 \mathrm{Myr}$ corresponding to the age of M 37 (Figure 14). We see that at this old age the period distribution mapping admits two branches of solutions, one with DR and another with SB rotation. This bimodality is caused by the very narrow range of the mapping at old ages which finally degenerates into a point at the solar age.

The second novelty of our investigation is that we have related the coupling time from the double-zone model to its corresponding value of the constant viscosity from our full stellar evolutionary model (Figure 7). In particular, the minimum coupling time of $30 \mathrm{Myr}$ obtained in our analysis of DR of solar-type stars roughly corresponds to $v_{0}=7.5 \times 10^{4} \mathrm{~cm}^{2} \mathrm{~s}^{-1}$, whereas the longest coupling time of $\sim 80$ Myr for stars with $M=1.0 \pm 0.1 M_{\odot}$ implies that in some of them the internal transport of angular momentum can be as slow as that modeled with a viscosity $v_{0} \approx 3 \times 10^{4} \mathrm{~cm}^{2} \mathrm{~s}^{-1}$. 
K-S Probability (NGC2264 to M37, M = 1.0 $\mathrm{M}_{\text {sun }}, \mathrm{P}_{\mathrm{c}}=1.5$ )
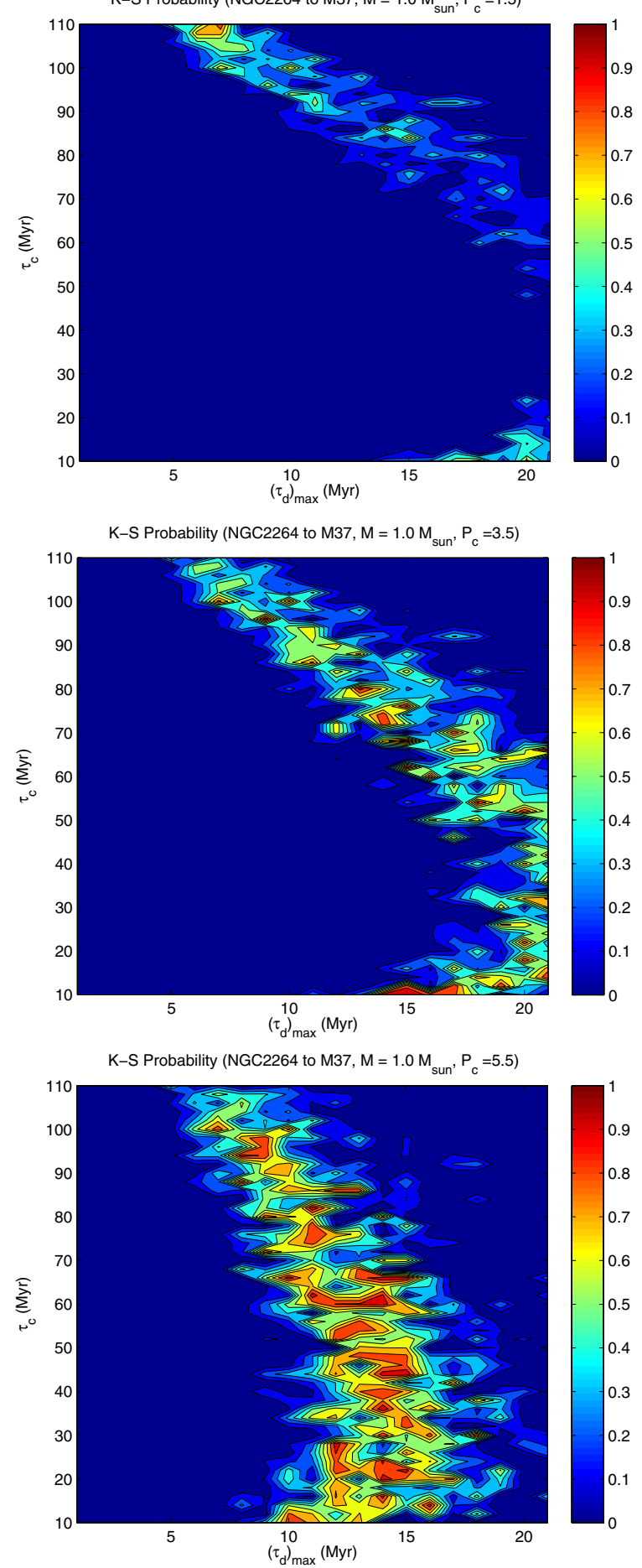

K-S Probability (NGC2264 to M37, M = 1.0 $\mathrm{M}_{\text {sun }}, \mathrm{P}_{\mathrm{c}}=2.5$ )

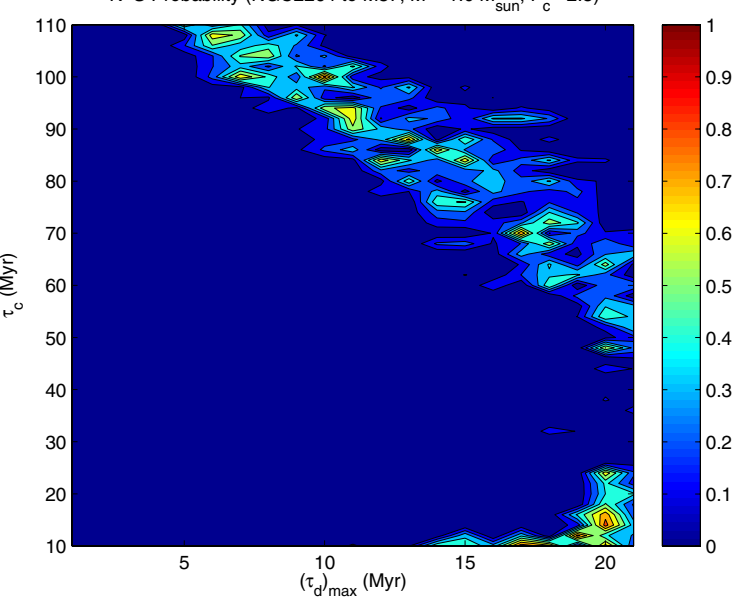

K-S Probability (NGC2264 to M37, M $=1.0 \mathrm{M}_{\text {sun }}, \mathrm{P}_{\mathrm{C}}=4.5$ )

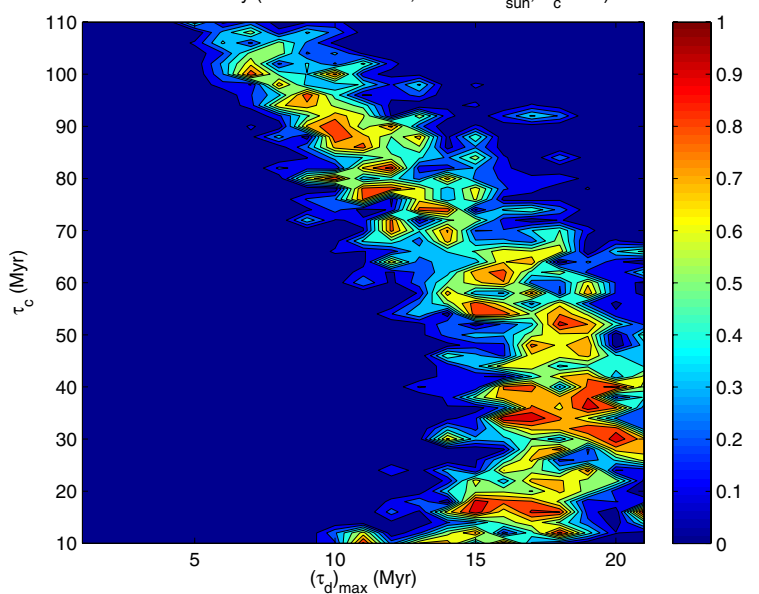

K-S Probability (NGC2264 to M37, M = 1.0 M $\mathrm{M}_{\text {sun }}, \mathrm{P}_{\mathrm{c}}=3.5$ )

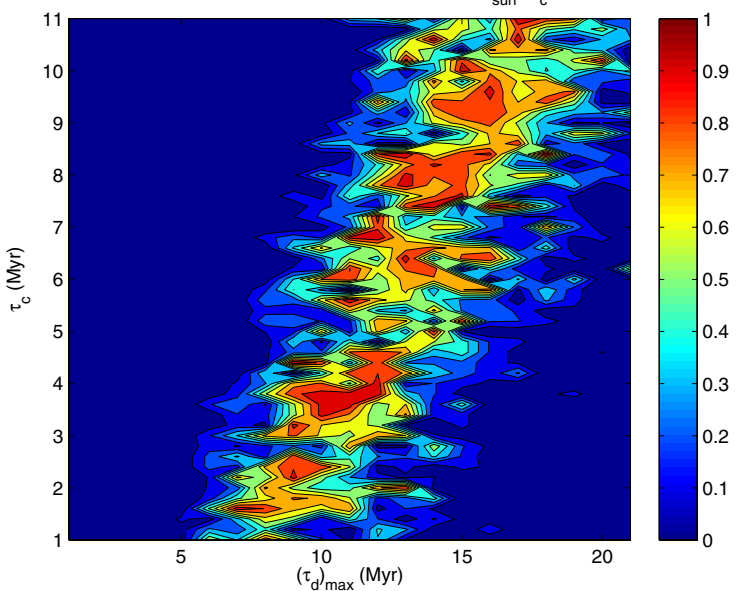

Figure 14. Contours of the Kolmogorov-Smirnov probability of the hypothesis that the observed and modeled period distributions of $\mathrm{M} 37$ stars with $M=1.0 \pm 0.1 M_{\odot}$ have been drawn from the same real distribution. The initial periods are taken from the NGC 2264 cluster. The double-zone model with the dependence (16) of $\tau_{\mathrm{c}}$ on $P_{0}$ has been employed. Simulations have been done for the shown intervals of the parameters $\tau_{\mathrm{c}}$ and $\left(\tau_{\mathrm{d}}\right)_{\max }$, and for the values of $P_{\mathrm{c}}$ specified in parenthesis atop each panel. Because of the convergence to the solar rotation, there is an ambiguity in the rotational evolution mode (SB or DR) for this $550 \mathrm{Myr}$ old cluster. (A color version of this figure is available in the online journal.)

Finally, we have shown that original Spruit's prescription always enforces SB rotation in a solar-type star, no matter how slowly it rotates. Therefore, it cannot be accepted as a physical mechanism for the internal angular momentum redistribution in radiative cores of solar-type stars many of which are indirectly proved to possess a degree of DR.
We have also found that the revised prescription for the Tayler-Spruit dynamo (Denissenkov \& Pinsonneault 2007) results in the rotational evolution superficially resembling that obtained using the original prescription, although the former leaves a large differentially rotating radiative core in the present-day solar model (Figure 6). Hence, the revised prescription appears 

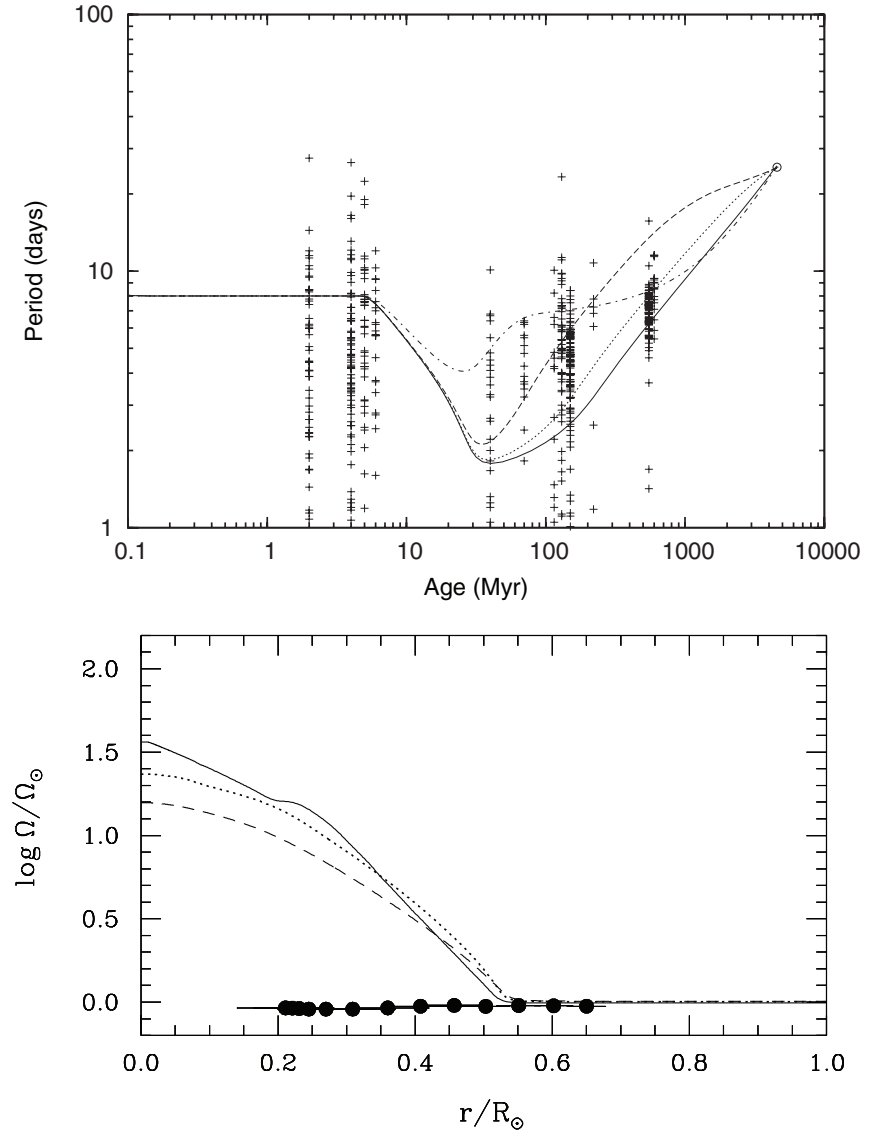

Figure 15. Rotation period evolution and final rotation profiles in the solar models computed using Spruit's revised prescription (solid curves) and the combined viscosity (Equation (18)) (dotted curves). Dashed curves represent results obtained with the combined viscosity in which the term $v_{\mathrm{GSF}}$ has been multiplied by a factor of 10 . For comparison, dot-dashed curve in upper panel shows the rotational evolution computed using the double-zone model with $\tau_{\mathrm{c}}=100 \mathrm{Myr}$ (a model with DR).

to be in conflict both with the rotation period data for solar-type stars in open clusters (like the original prescription) and with the helioseismic data (unlike the original prescription). It is worth seeing if other possible angular momentum transport mechanisms can assist the revised prescription in bringing the rotating solar model closer to observations. To test this idea, we have supplemented the effective magnetic viscosity (Equation (12)) with viscosities arising from the secular shear instability (denoted by the subscript "ss" below), Goldreich-Schubert-Fricke instability (GSF), as well as with the molecular viscosity (mol) and a viscosity $v_{\mathrm{mc}}=|r U(r)| / 5$ that approximately describes the transport of angular momentum by meridional circulation as a diffusion process:

$$
v=\left(v_{\mathrm{e}}\right)_{\mathrm{min}}^{(12)}+v_{\mathrm{ss}}+v_{\mathrm{GSF}}+v_{\mathrm{mc}}+v_{\mathrm{mol}} \text {. }
$$

To calculate the quantities $v_{\mathrm{ss}}, v_{\mathrm{GSF}}$, and the meridional circulation velocity $U(r)$, we have used the corresponding equations from the Appendix of Chanamé et al. (2005). Note that their expression for $U(r)$ neglects terms depending on the $\mu$-gradient and its derivatives. Therefore, the meridional circulation in this approximation is expected to penetrate deeper into the radiative core than in the case of its fully consistent implementation originally proposed by Maeder \& Zahn (1998) that had later been applied to study the rotational mixing in solar-metallicity MS stars with $M \geqslant 1.35 M_{\odot}$ by Palacios et al. (2003).
In Figure 15, the dotted curves represent results of our computations with the combined viscosity (Equation (18)) substituted into Equation (1). Note that a slightly increased amount of angular momentum that can be transported from the core to the envelope on a longer hydrodynamical timescale has changed our results in the right directions: first, after the wind constant $K_{\mathrm{W}}$ is properly recalibrated, the $P$-age relation has shifted toward longer periods, and, second, the degree of DR in the core has slightly been reduced. To find out if these changes will further grow in the same directions when the efficiency of supplementary angular momentum transport mechanisms increases, we have multiplied $v_{\mathrm{GSF}}$, the dominating viscosity in the core, by a factor of 10 . Results of this artificial viscosity enhancement are plotted in Figure 15 with dashed curves. We see that both the $P$-age relation and the core rotation profile have indeed continued to change in the right directions. This exercise shows that the revised prescription cannot be rejected as easily as the original one. There remains a possibility that, when being assisted by other transport processes, it may reproduce the observational data yet.

Our main conclusion is critically based on a comparison of theoretical predictions and observations of rotation periods for the slowest rotators in the intermediate-age open clusters. Therefore, if the observational data were biased toward the longest periods that would undermine confidence in our results. The real situation turns out to be opposite. Observations for some of our used open clusters (for those with smaller data samples) are actually biased toward the shortest periods, as is expected from the photometric period measurement procedure that needs longer observational times to accumulate data sufficient for extracting longer periods. The following example illustrates this bias. Filled circles in upper panels of Figure 16 represent the Pleiades $v \sin i$ unbiased data from Terndrup et al. (2000). For comparison, open circles in the upper left panel show the Pleiades data used in our work that do appear to be biased toward lower rotation periods. On the other hand, open circles in the upper right panel are the M 35 data from Meibom et al. (2009). Their original periods have been transformed into $v \sin i$ values using a randomly generated angle $0 \leqslant i \leqslant \pi / 2$ and $R=R_{\odot}$. The lower right panel shows that the unbiased data for the two clusters of similar age look alike $\left(P_{\mathrm{KS}}=0.112\right.$, the medians are 6.8 and 8.5, the first and third quartiles are 5.4 and 13 for the Pleiades, and 4.7 and 18 for M 35). In contrast, the biased Pleiades data in the lower left panel have very different median and third quartile values, 15 and 39, respectively.

To sum up, our main conclusions can concisely be formulated as follows. Whereas the period distributions for the fastest rotators among solar-type stars in open clusters are very well reproduced assuming their SB rotation, the period distributions for the slowest rotators are better described by stellar models with DR in their radiative cores. This conclusion is in agreement with previous results reported by Irwin et al. (2007). Our new result is that the original prescription for the Tayler-Spruit dynamo always enforces SB rotation in a solar-type star even if the star is a slow rotator. Therefore, this angular momentum transport mechanism is unlikely to operate in solar-type early MS stars. The revised prescription for the Tayler-Spruit dynamo cannot explain the observed period distributions either. Besides, it leaves a large rapidly rotating radiative core in the presentday solar model. To be consistent with observations, the revised prescription needs to be assisted by other angular momentum transport mechanisms that must be able to penetrate into the deep core and operate on a longer timescale. 

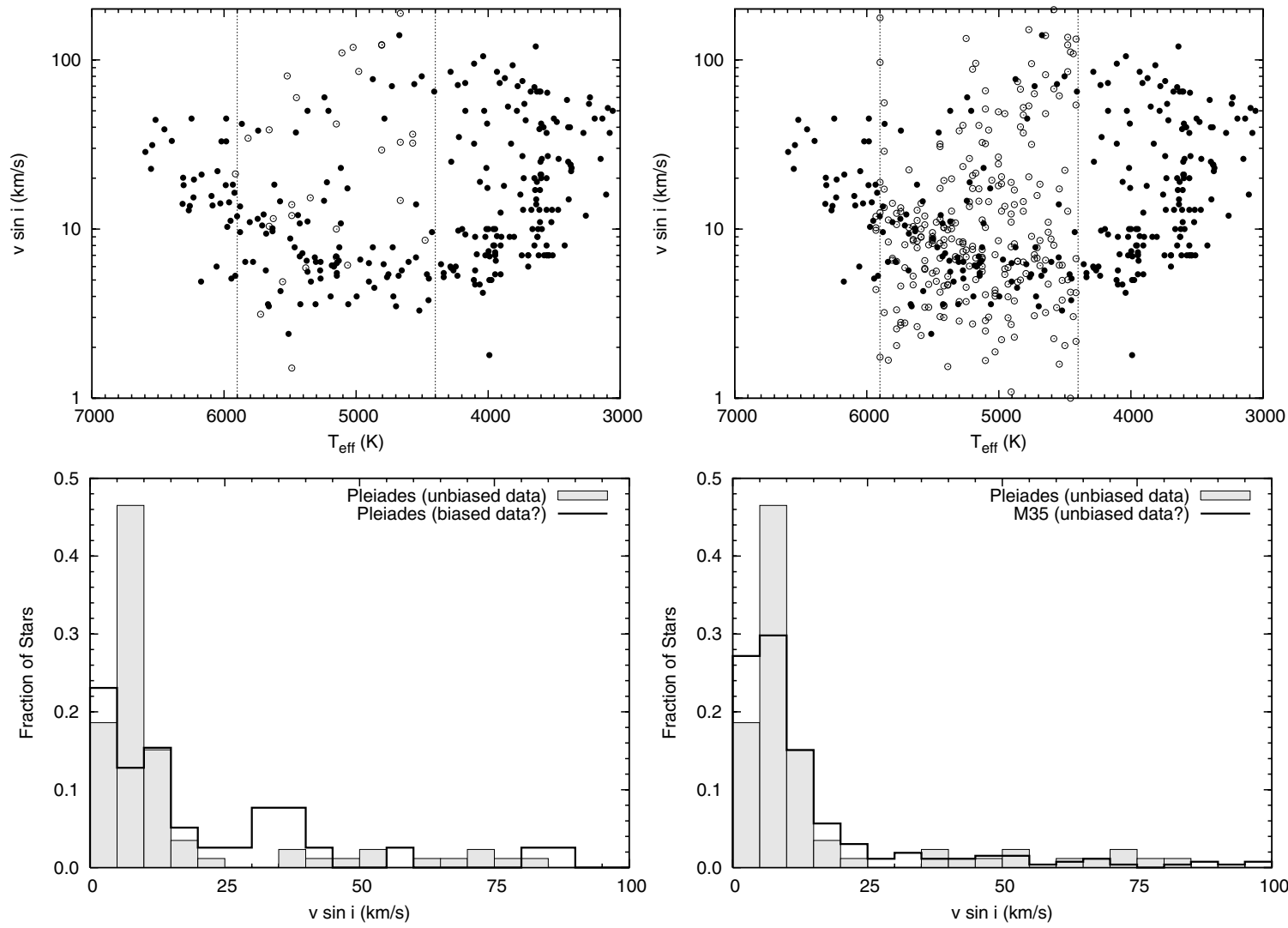

Figure 16. Filled circles in upper panels are the Pleiades $v \sin i$ unbiased data from Terndrup et al. (2000). Open circles in the upper left panel are the Pleiades data used in this paper that appear to be biased toward lower rotation periods. Open circles in the upper right panel are the M 35 data from Meibom et al. (2009). Periods have been transformed into $v \sin i$ values using a randomly generated angle $0 \leqslant i \leqslant \pi / 2$ and $R=R_{\odot}$. The lower right panel shows that the unbiased data for the two clusters of similar age look alike $\left(P_{\mathrm{KS}}=0.112\right.$, the medians are 6.8 and 8.5, the first and third quartiles are 5.4 and 13 for the Pleiades, and 4.7 and 18 for $\left.\mathrm{M} 35\right)$. In contrast, the biased Pleiades data in the lower left panel have very different median and third quartile values, 15 and 39 , respectively. Vertical dotted lines in upper panels show the range of $0.7 \lesssim M / M_{\odot} \lesssim 1.1$.

We are grateful to Luisa Rebull for providing us with the latest data on rotation periods of open cluster low-mass stars. We acknowledge support from the NASA grant NNG05 GG20G.

\section{REFERENCES}

Alexander, D. R., \& Ferguson, J. W. 1994, ApJ, 437, 879

Allain, S. 1998, A\&A, 333, 629

Andronov, N., Pinsonneault, M. H., \& Sills, A. 2003, ApJ, 582, 358

Angulo, C., et al. 1999, Nucl. Phys. A, 656, 3

Bouvier, J., Forestini, M., \& Allain, S. 1997, A\&A, 326, 1023

Bouwman, J., Lawson, W. A., Dominik, C., Feigelson, E. D., Henning, T. Tielens, A. G. G. M., \& Waters, L. B. F. M. 2006, ApJ, 635, L57

Chanamé, J., Pinsonneault, M., \& Terndrup, D. M. 2005, ApJ, 631, 540

Charbonneau, P., \& MacGregor, K. B. 1993, ApJ, 417, 762

Charbonnel, C., \& Talon, S. 2005, Science, 309, 2189

Couvidat, S., García, R. A., Turck-Chièze Corbard, T., Henney, C. J., \& JiménezReyes, S. 2003, ApJ, 597, L77

Damjanov, I., Jayawardhana, R., Scholz, A., Ahmic, M., Nguyen, D. C., Brandeker, A., \& van Kerkwijk, M. H. 2007, ApJ, 670, 1337

Denissenkov, P. A. 2010, arXiv:1002.2261v1

Denissenkov, P. A., \& Pinsonneault, M. 2007, ApJ, 655, 1157

Denissenkov, P. A., Pinsonneault, M., \& MacGregor, K. B. 2008, ApJ, 684, 757

Denissenkov, P. A., \& VandenBerg, D. A. 2003, ApJ, 598, 1246

Eggenberger, P., Maeder, A., \& Meynet, G. 2005, A\&A, 440, L9

García, R. A., Turck-Chièze, S., Jiménez-Reyes, S. J., Ballot, J., Pallé, P. L., Eff-Darwich, A., Mathur, S., \& Provost, J. 2007, Science, 316, 159

Grevesse, N., \& Noels, A. 1993, in Origin and Evolution of the Elements, ed. N. Prantzos, E. Vangioni-Flam, \& M. Casse (Cambridge: Cambridge Univ. Press), 15

Hartman, J. D., et al. 2009, ApJ, 691, 342
Hillenbrand, L. A. 2005, arXiv:astro-ph/0511083v1

Irwin, J., Aigrain, S., Bouvier, J., Hebb, L., Hodgkin, S., Irwin, M., \& Moraux, E. 2009, MNRAS, 392, 1456

Irwin, J., Aigrain, S., Hodgkin, S., Irwin, M., Bouvier, J., Clarke, C., Hebb, L. \& Moraux, E. 2006, MNRAS, 370, 954

Irwin, J., Hodgkin, S., Aigrain, S., Bouvier, J., Hebb, L., Irwin, M., \& Moraux, E. 2008a, MNRAS, 384, 675

Irwin, J., Hodgkin, S., Aigrain, S., Bouvier, J., Hebb, L., \& Moraux, E. 2008b, MNRAS, 383, 1588

Irwin, J., Hodgkin, S., Aigrain, S., Hebb, L., Bouvier, J., Clarke, C., Moraux, E., \& Bramich, D. M. 2007, MNRAS, 377, 741

Itoh, N., Hayashi, H., Nishikawa, A., \& Kohyama, Y. 1996, ApJS, 102, 411

Jayawardhana, R., Coffey, J., Scholz, A., Brandeker, A., \& van Kerkwij, M. H. 2006, ApJ, 648, 1206

Keppens, R., MacGregor, K. B., \& Charbonneau, P. 1995, A\&A, 294, 469

Koenigl, A. 1991, ApJ, 370, L39

Krishamurthi, A., Pinsonneault, M. H., Barnes, S., \& Sofia, S. 1997, ApJ, 480, 303

Krishamurthi, A., et al. 1998, ApJ, 493, 914

Lyo, A.-R., \& Lawson, W. A. 2005, J. Korean Astron. Soc., 38, 24

MacGregor, K. B. 1991, in Angular Momentum Evolution of Young Stars, ed. S. Catalano \& J. R. Stauffer (Dordrecht: Kluwer), 315

MacGregor, K. B., \& Brenner, M. 1991, ApJ, 376, 204

Maeder, A., \& Zahn, J.-P. 1998, A\&A, 334, 1000

Mamajek, E. E. 2009, in AIP Conf. Proc. 1158, Exoplanets and Disks: Their Formation and Diversity, ed. T. Usuda, M. Tamura, \& M. Ishi (Melville, NY: AIP), 3

Matt, S., \& Pudritz, R. E. 2005, ApJ, 632, L135

Meibom, S., Mathieu, R. D., \& Stassun, K. G. 2009, ApJ, 695, 679

Mestel, L., \& Weiss, N. O. 1987, MNRAS, 226, 123

Palacios, A., Talon, S., Charbonnel, C., \& Forestini, M. 2003, A\&A, 399, 603

Palla, F., \& Stahler, S. W. 1991, ApJ, 375, 288 
Pinsonneault, M. H., Kawaler, S. D., \& Demarque, P. 1990, ApJS, 74, 501

Pinsonneault, M. H., Kawaler, S. D., Sofia, S., \& Demarque, P. 1989, ApJ, 338, 424

Prosser, C. F., et al. 1995, PASP, 107, 211

Radick, R. R., Thompson, T. D., Lockwood, G. W., Duncan, D. K., \& Baggett, W. E. 1987, ApJ, 321, 459

Rebull, L. M., Wolff, S. C., \& Strom, S. E. 2004, AJ, 127, 1029

Rogers, F. J., \& Iglesias, C. A. 1992, ApJ, 401, 361

Rüdiger, G., Gellert, M., \& Schultz, M. 2009, MNRAS, 399, 996

Scholz, A., \& Eislöffel, J. 2007, MNRAS, 381, 1638

Shu, F., Najita, J., Ostriker, E., Wilkin, F., Ruden, S., \& Lizano, S. 1994, ApJ, 429, 781

Sicilia-Aguilar, A., et al. 2009, ApJ, 701, 1188
Siess, L., \& Livio, M. 1997, ApJ, 490, 785

Sills, A., Pinsonneault, M. H., \& Terndrup, D. M. 2000, ApJ, 534, 335

Skumanich, A. 1972, ApJ, 171, 565

Spruit, H. C. 1999, A\&A, 349, 189

Spruit, H. C. 2002, A\&A, 381, 923

Tayler, R. J. 1973, MNRAS, 161, 365

Terndrup, D. M., Stauffer, J. R., Pinsonneault, M. H., Sills, A., Yuan, Y., Jones, B. F., Fischer, D., \& Krishnamurthi, A. 2000, AJ, 119, 1303

Tinker, J., Pinsonneault, M., \& Terndrup, D. M. 2002, ApJ, 564, 877

Tomczyk, S., Schou, J., \& Thompson, M. J. 1995, ApJ, 448, L57

Weber, E. J., \& Davis, L., Jr. 1967, ApJ, 148, 217

Zahn, J.-P. 1992, A\&A, 256, 115

Zahn, J.-P., Brun, A. S., \& Mathis, S. 2007, A\&A, 474, 145 